Check for updates

Cite this: RSC Adv., 2017, 7, 26809

\title{
Effect of dietary oxidized tyrosine products on insulin secretion via the oxidative stress-induced mitochondria damage in mice pancreas
}

\author{
Yin-Yi Ding, (D) ${ }^{a}$ Xiang-Rong Cheng, ${ }^{a}$ Zhu-Qing Li, ${ }^{a}$ Sha-Ji Wu, ${ }^{a}$ Yuhui Yang, ${ }^{a}$ \\ Yong-Hui Shi ${ }^{\text {ab }}$ and Guo-Wei Le ${ }^{\star a b}$
}

Oxidized tyrosine products (OTPs) have been detected in commercial food and have been demonstrated to induce oxidative damage in vivo. The pancreas plays an important role in glucose metabolism, but its antioxidant capacity is low. The present study investigates the potential impact of dietary OTPS on the pancreatic function. Sprague Dawley (SD) rats (8 rats per group) were fed a diet containing OTPs for 24 weeks, and the blood glucose and plasma insulin levels were then measured. Elevated fasting blood glucose and decreased plasma insulin levels indicated dysfunction of the pancreatic islets. The rats fed the OTPs-containing diet also exhibited pancreatic oxidative stress, accompanied by inflammation. Furthermore, the expression of genes involved in the Nrf2/ARE pathway was down-regulated in the OTPs-treated groups. In addition, supplementation with lipoic acid (LA) significantly remitted the OTPS induced oxidative stress of the pancreas, and mitigated the effects of OTPs on the blood glucose and insulin synthesis. A gavage experiment was performed to explore whether dityrosine (Dityr), a major component of OTPs, was responsible for the injury induced by OTP. The OTPs, including Dityr, induced mitochondrial defects in cultured mice insulinoma MIN- 6 cells and mice pancreas, as evidenced by less ATP production, loss of mitochondrial membrane potential, mitochondrial DNA (mtDNA) depletion, and alteration of the mRNA levels of genes involved in mitochondrial function. Moreover, the apoptosis of pancreatic islets and MIN-6 cells increased after exposure to OTPs/Dityr. The findings suggest that decreased insulin secretion triggered by OTPs may be mediated by oxidative stress and mitochondrial damage in pancreatic $\beta$ cells.

Received 11th March 2017 Accepted 25th April 2017 DOI: $10.1039 / c 7 r a 02945 d$

rsc.li/rsc-advances of milk containing oxidized protein has negative effects on children, including lower growth rates, eczema and dry stool. ${ }^{12,13}$ In previous studies, we verified that long-term exposure to oxidized casein or oxidized tyrosine impaired the antioxidant defense system and induced hepatic and renal fibrosis. ${ }^{14,15}$ Previous research also suggested that Dityr could induce oxidative damage in the brain, leading to novel object recognition deficits. ${ }^{\mathbf{1 6}}$

Given the findings of the aforementioned studies, examining the relationship between oxidized proteins and oxidative stress is presently a major research topic. Redox status is crucial for normal metabolism. However, the pancreas inherently contains only a fraction of the antioxidant enzymatic activities, in comparison to the liver. ${ }^{17}$ As result, the pancreas may be a major target of oxidative stress in the condition of diabetes. In patients with diabetes, the activities of antioxidant enzymes in the plasma are weaker than those of individuals without diabetes. ${ }^{18}$ The influence of oxidative damage induced by oxidized proteins on the bio-function of pancreatic $\beta$ cells has not been fully explored.

The mitochondrion is a key organelle in $\beta$ cells, which is crucial for ATP generation and other factors that link glucose metabolism to insulin secretion. ${ }^{19-21}$ However, mitochondria
${ }^{a}$ Food Nutrition and Functional Factors Research Center, School of Food Science and Technology, Jiangnan University, Wuxi, 214122, China

${ }^{b}$ The State Key Laboratory of Food Science and Technology, School of Food Science and Technology, Jiangnan University, Lihu Avenue 1800, Wuxi 214122, China. E-mail: lgw@jiangnan.edu.cn; Fax: +86 5108591 7789; Tel: +86 51085917789 
are susceptible to oxidative damage, and excessive generation of reactive oxygen species (ROS) can lead to mitochondrial dysfunction..$^{22,23}$ The activities of antioxidant enzymes are largely responsible for the protection of mitochondria against ROS. ${ }^{24}$ Although a number of stimuli modulate the synthesis and release of insulin, mitochondrial oxidative metabolism is the most important factor in glucose-stimulated insulin secretion. ${ }^{25}$

In the present study, we investigate the effects of OTPs on insulin secretion and the redox status of the pancreas. Since Dityr accounts for $22 \%$ of OTPs, ${ }^{26}$ the potential role of Dityr in pancreatic injury induced by OTPs was studied in a 10 week gavage experiment involving C57BL/6 mice administered with OTPs or pure Dityr. This study also examines whether exposure to Dityr results in mitochondrial dysfunction, subsequently attenuated insulin secretion and ultimately, triggered hyperglycemia.

\section{Materials and methods}

\subsection{Oxidized tyrosine products preparation}

OTP was prepared by our laboratory according to the method of Kurahashi, ${ }^{27}$ with a little modification, as previously described. ${ }^{26}$ Briefly, L-tyrosine was dispersed in $0.05 \mathrm{M}$ phosphate buffer ( $\mathrm{pH} 7.4$ ) with the concentration of $1 \mathrm{mg} \mathrm{mL}^{-1}$. The tyrosine solution was added to $\mathrm{H}_{2} \mathrm{O}_{2}(5 \mathrm{mM})$ and $\mathrm{CuSO}_{4}(0.05$ $\mathrm{mM})$ and mixed in sealed tubes. The mixtures were shaken at $60 \mathrm{rpm}$ in a thermostatic bath at $45 \pm 0.1{ }^{\circ} \mathrm{C}$ for $2 \mathrm{~h}$. At the end of the reaction, $\mathrm{Cu}^{2+}$ was removed using cation exchange resin. Freeze-drying was applied to remove the remaining hydrogen peroxide and dry the OTPs sample. The major constituents of OTPs were assessed in the previous study in our lab and it was found that the OTPs sample mainly contained Dityr, the content of which was $22 \%(\mathrm{w} / \mathrm{w}){ }^{26}$

\subsection{4 week dietary OTPs experiment in SD rats}

2.2.1. Animals and treatment. Male Sprague Dawley (SD) rats (weighing $300 \pm 20 \mathrm{~g}$ ) were purchased from the Model Animal Research Center of Nanjing University. Rats were housed in individual cages in a temperature-controlled room at $24 \pm 2{ }^{\circ} \mathrm{C}$, with a $12 \mathrm{~h}$ light-dark cycle (Research animal center of Jiangnan University).

After 7 days of acclimatization, SD rats were divided into 5 groups $(n=8)$ :

Control group (rats received the standard diet);

OTP1 group (rats received the standard diet together with $4 \mathrm{~g}$ $\mathrm{kg}^{-1}$ of OTPs);

OTP2 group (rats received the standard diet together with $8 \mathrm{~g}$ $\mathrm{kg}^{-1}$ of OTPs);

OTP + LA group (rats received $8 \mathrm{~g} \mathrm{~kg}^{-1}$ of OTPs and $2 \mathrm{~g} \mathrm{~kg}^{-1}$ of lipoic acid (LA)).

Tyr group (rats received the standard diet together with $8 \mathrm{~g}$ $\mathrm{kg}^{-1}$ of tyrosine).

The dose of OTPs we used in this experiment was consistent with the previous studies in our lab. ${ }^{26}$ All animal treatments were performed in accordance with the ethical standards laid down in the guidelines of National Institutes of Health Guide for the Care and Use of Laboratory Animals (China) and in the 1964 Declaration of Helsinki and its later amendments. All animal studies were approved by the Laboratory Animals Ethics Committee of Jiangnan University.

2.2.2. Body weight, food intake, blood glucose, glucose tolerance test, plasma insulin content. The body weight was measured weekly. The $24 \mathrm{~h}$ food intake was measured using the Comprehensive Laboratory Animal Monitoring System (CLAMS, Columbus Instruments, Columbus, $\mathrm{OH}$ ) at the end of the 24 week experimental period. For blood glucose tests, rats were fasted overnight for $12 \mathrm{~h}$, and blood samples were obtained from the tail vein. Animals were then given $2 \mathrm{~g} \mathrm{~kg}^{-1}$ body weight of glucose by gavage, and blood samples were taken at the indicated intervals $(0,10,30,60,90,120 \mathrm{~min}$ after gavage). Blood glucose was tested using One Touch Sure Step test strips (Lifescan, Milpitas, California, U.S.A.). Plasma insulin concentration was measured using the rat ELISA kits (Huijia biotechnology, Xiamen, P. R. China) according to the manufacturer's instruction.

2.2.3. Oxidative stress biomarkers and activities of antioxidant enzymes. After a 24 week period, the rats were sacrificed. Reactive oxygen species (ROS) in the blood were measured by an electron-spin resonance spectrometer system (ESR) $\left(\mathrm{EMX}^{\mathrm{plus}}\right.$ 10/12, Bruker, Germany) using 5,5-dimethyl-1-pyrroline-Noxide (DMPO) (Sigma-Aldrich Corp., St. Louis, MO, U.S.A.) as the ROS trapping agent. The signal obtained was used to calculate the central peak height of the ESR signal (the value of the top point minus the value of the bottom point).

The activity of superoxide dismutase (SOD), catalase (CAT), glutathione peroxidation (GPx), and malondialdehyde (MDA) levels in the pancreas and total antioxidants (T-AOC), reduced glutathione (GSH), oxidized glutathione (GSSG) in the blood were assayed by corresponding kits (Jiancheng Bioengineering Institute, Nanjing, P. R. China). Total protein contents were determined by the method of Lowry et al., ${ }^{28}$ using bull serum albumin (BSA) as the protein standard. The levels of tumor necrosis factor- $\alpha$ (TNF- $\alpha$ ), interleukin-6 (IL-6) in the plasma, Dityr and 3-nitrotyrosine (3-NT) in the pancreas were measured using the rat ELISA kits (Huijia Biotechnology, Xiamen, P. R. China), according to the manufacturer's instructions.

\subsection{0 week gavage experiment using OTPs and Dityr in C57BL/6 mice}

2.3.1. Animal treatment. The major constituent of OTPs is Dityr, so we performed an additional Dityr gavage experiment to explore whether Dityr was responsible for the injury induced by OTPs. Since the preparation of Dityr is very costly and timeconsuming, we chose to use mice (with body mass much lower than that of rats) for this gavage study, and 4 week-old male C57BL/6 mice were purchased from the Model Animal Research Center of Nanjing University, China. The mice were housed in a temperature-controlled room at $24 \pm 2{ }^{\circ} \mathrm{C}$ with a $12 \mathrm{~h}$ light-dark cycle, with free access to food and water. Mice were randomly divided into three groups $(n=8)$, which received Dityr (320 $\mu \mathrm{g}$ per kg per bw per day) (Dityr group), OTPs (1450 $\mu \mathrm{g}$ 
per $\mathrm{kg}$ per bw per day) (OTPs group), and saline solution (control group). The dose of Dityr used in the animal experiment was consistent with the previous studies in our lab. ${ }^{16}$ The mass percent of Dityr in the OTPs sample was $22 \%$; the effect of $320 \mu \mathrm{g}$ per $\mathrm{kg}$ per bw per day Dityr corresponds to that of 1450 $\mu \mathrm{g}$ per kg per bw per day OTPs. All animal studies were performed in accordance with the ethical standards laid down in the Guidelines for Laboratory Animals Care, Jiangsu Province (China) and in the 1964 Declaration of Helsinki and its later amendments.

2.3.2. Histopathological analysis. The pancreas was fixed in $10 \%$ formaldehyde phosphate buffer for $24 \mathrm{~h}$, and embedded in paraffin. Hematoxylin and Eosin (HE) staining was performed on $5 \mu \mathrm{m}$ serial sections to analyze the histopathological changes in the pancreatic islets. Islet cell apoptosis was determined by terminal deoxynucleotidyl transferase dUTPnick end labeling (TUNEL) staining (Beyotime Biotechnology, Shanghai, China). The pictures of the slices were taken by optical microscope (BX41, Olympus, Tokyo, Japan). Images were analyzed using the Image-Pro Plus 5.0 software (Media Cybernetics Inc., Rockville, U.S.A.).

\subsection{Cell experiment using mice MIN-6 cells}

2.4.1. Culture and cell treatment. The MIN- 6 cells derived from mice insulinoma were purchased from the cell bank of the Type Culture Collection of Chinese Academy of Sciences (Shanghai, China) and were grown in DMEM medium supplemented with $4.0 \mathrm{mM}$ glucose and $10 \%$ fetal bovine serum (Gibco, South Logan, UT, USA). Cells were seeded in plates and permitted to adhere at $37{ }^{\circ} \mathrm{C}$ in a humidified atmosphere containing $95 \%$ air and $5 \% \mathrm{CO}_{2}$ for $24 \mathrm{~h}$. When the cells reached $40 \%$ confluence, they were stimulated with Dityr in a final concentration of $0,0.01,0.1,1$ $\mu \mathrm{M}$ for $72 \mathrm{~h}$. In addition, we also set a positive control group, and cells were stimulated with $\mathrm{H}_{2} \mathrm{O}_{2}$ in a final concentration of $200 \mu \mathrm{M}$ for $72 \mathrm{~h}$.

2.4.2. Cell viability assay. At the end of the treatment, MTT $\left(0.5 \mathrm{mg} \mathrm{mL} \mathrm{m}^{-1}\right)$ was added to each well for $4 \mathrm{~h}$. Subsequently, medium and MTT were removed, and the precipitate was dissolved with DMSO. The optical density was measured at $570 \mathrm{~nm}$ with Multiskan Spectrum (Epoch, BioTek, Vermont, U.S.A.).

2.4.3. Glucose-stimulated insulin secretion (GSIS) assay. After the treatment, the medium containing Dityr or $\mathrm{H}_{2} \mathrm{O}_{2}$ was discarded and phosphatic buffer solution was used for gently washing the cells twice. The cells were then pre-incubated with Kreb-Ringer bicarbonate HEPES (KRBH) buffer for $1 \mathrm{~h}$. Afterward, the cells were incubated in KRBH buffer containing 2.5 or $16.7 \mathrm{mM}$ glucose for $1 \mathrm{~h}$. Insulin concentrations were measured using mice insulin ELISA kits (Huijia Biotechnology, Xiamen, P. R. China). The levels of secreted insulin were normalized to the protein content, which was determined with the BCA Protein Assay Kit (Beyotime Biotechnology, Shanghai, P. R. China).

2.4.4. Measurement of cellular ROS. Cellular oxidative stress due to ROS generation by Dityr and $\mathrm{H}_{2} \mathrm{O}_{2}$ was determined with a fluorescence probe, DCFH-DA. The DCF fluorescence intensity is believed to be parallel to the intracellular ROS
Table 1 Primer's sequences of SD rats for qRT-PCR

\begin{tabular}{lll}
\hline Gene & Forward $\left(5^{\prime}\right.$ to $\left.3^{\prime}\right)$ & Reverse $\left(5^{\prime}\right.$ to $\left.3^{\prime}\right)$ \\
\hline Pdx-1 & TCTGCCTCTGGGA & TCGCCTGGTGG \\
& CTCTTTC & CTGTTAT \\
Mafa & TCCTCGCTCATTT & GGTAAGTTCCTC \\
& GCTCTG & GGCTTCCT \\
Glut 2 & TTTGGGTGTCCT & CGTCTGGTGTCG \\
& CTGGATG & ACGATGTTG \\
Gck & TCACCTTCTCCTTC & CTTCTGC \\
& CCTGTG & TCTTGCTGCTTCC \\
Creb & GGAGTTGTTATGGC & CTGTTCT \\
& GTCCTC & GCTCAGGATTGGT \\
Nrf2 & TTCAGCCAGGCTATA & GGTATGAGA \\
& AGTCAGG & AAAGCCCTACAGC \\
Ho-1 & AAGACTGCGTTCCTG & AACTGTCG \\
& CTCAAC & GAGGGACGCCAC \\
Gpx-1 & CAGTCGGTGTATGCC & ATTCTCG \\
& TTCTCG & GGGGTGTTGAAG \\
$\beta$-Actin & TGTCACCAACTGGGA & GTCTCAAA \\
& CGATA &
\end{tabular}

formation. At the end of treatment, the medium containing Dityr or $\mathrm{H}_{2} \mathrm{O}_{2}$ was discarded and phosphate buffer solution was used to gently wash cells twice and then DCFH-DA, at the final concentration of $10 \mu \mathrm{M}$, was incubated for $30 \mathrm{~min}$ in the dark.

Table 2 Primer's sequences of C57BL/6 mice and MIN- 6 cells for qRT-PCR

\begin{tabular}{|c|c|c|}
\hline Gene & Forward $\left(5^{\prime}\right.$ to $\left.3^{\prime}\right)$ & Reverse $\left(5^{\prime}\right.$ to $\left.3^{\prime}\right)$ \\
\hline \multirow[t]{2}{*}{ Glut2 } & ACCCCACTTACAG & CACAGACAGAGACC \\
\hline & TCACACCA & AGAGCATAG \\
\hline \multirow[t]{2}{*}{ Gck } & AGGAGGCCAGTGT & CTCCCAGGTCTAAG \\
\hline & AAAGATGT & GAGAGAAA \\
\hline \multirow[t]{2}{*}{ TFAM } & ATCCCCTCGTCTATC & TTCTGCTTCTGGTA \\
\hline & AGTCTTGTCT & GCTCССTC \\
\hline \multirow[t]{2}{*}{ Citrate synthase } & GGACAATTTTCCAAC & AGTCAATGGCTCC \\
\hline & CAATCTGC & GATACTGC \\
\hline \multirow[t]{2}{*}{ ATP6 } & AGGATTCCCAATCGT & CCTTTTGGTGTGTG \\
\hline & TGTAGCC & GATTAGCA \\
\hline \multirow[t]{2}{*}{$U C P 2$} & CACGCAGCCTCTACAATG & TCAGCACAGTTGA \\
\hline & & CAATGG \\
\hline \multirow[t]{2}{*}{$O G D H$} & GTTTCTTCAAACGTG & GCATGATTCCAGGG \\
\hline & GGGTTCT & GTCTCAAA \\
\hline \multirow[t]{2}{*}{ Kir6.2 } & TGGGTGGTAACGG & GGTGCAGGTCA \\
\hline & CATCTTC & CTAGGAGC \\
\hline \multirow[t]{2}{*}{ Sur } & CAAGGTGTCCTCAAC & CCAGGTGCTATGG \\
\hline & AACGG & TGAATGTG \\
\hline \multirow[t]{2}{*}{$N D 2$} & CACGATCAACTGAA & ACGATGGCCAGGA \\
\hline & GCAGCAA & GGATAATT \\
\hline \multirow[t]{2}{*}{$\beta 2 M$} & TGTCAGATATGTCC & TGCTTAACTCTGCA \\
\hline & TTCAGCAAGG & GGCGTATG \\
\hline \multirow[t]{2}{*}{ Bax } & CCAGGATGCGTCC & GCAAAGTAGAAGAG \\
\hline & ACCAAGA & GGCAACCAC \\
\hline \multirow[t]{2}{*}{ Caspase 3} & CTGGAGAAATTCA & TGAGCATGGACACA \\
\hline & AAGGACGGG & ATACACGG \\
\hline \multirow[t]{2}{*}{$B c l-2$} & TCTTTGAGTTCGG & AGACAGCCAGGAGA \\
\hline & TGGGGTCAT & AATCAAACAGA \\
\hline \multirow[t]{2}{*}{$\beta$-Actin } & TGTCACCAACTG & GGGGTGTTGAAG \\
\hline & GGACGATA & GTCTCAAA \\
\hline
\end{tabular}


The DCF fluorescence intensity was detected using a fluorescence microplate reader (M5, Molecular Device, SVSE, USA) at an excitation wavelength of $488 \mathrm{~nm}$ and an emission wavelength of $525 \mathrm{~nm}$.

2.4.5. ATP generation analysis. At the end of treatment, the cells were collected, and the cellular ATP concentration was determined using an ATP assay kit (Jiancheng Bioengineering Institute, Nanjing, P. R. China), following the manufacturer's instructions.

\subsection{Mitochondrial DNA copy number analysis}

Total DNA of cells and tissues was extracted with Trizol reagent according to the manufacturer's protocol (Applied Biosystems, Foster City, CA, USA). The concentration of total DNA in each sample was quantified using a NanoDrop Spectrophotometer (ND2000, Thermo, Waltham, MA, USA). Relative quantification of mt-ND2 (mitochondrial DNA, mtDNA) and control n $\beta 2 \mathrm{M}$ (nuclear DNA, nDNA) was conducted in MIN-6 cells and the pancreas of C57BL/ 6 mice, based on a qRT-PCR protocol. The ratio of mtDNA/ nDNA is the indicator of the mtDNA copy number. ${ }^{29}$

\subsection{Determination of mitochondrial membrane potential}

The mitochondrial membrane potential in MIN-6 cells and the pancreas of C57BL/6 mice was measured using 5,5',6,6'-tetrachloro-1,1'3,3'-tetra-ethylimidacarbocyanine iodide fluorescent probe (JC-1) (Beyotime Biotechnology, Shanghai, P. R. China). The mitochondrial membrane potential loss was reflected by the increased green fluorescence from JC-1 monomers as well as the reduction of red fluorescence from JC- 1 aggregates. The mitochondrial membrane potential in the pancreas of $\mathrm{C} 57 \mathrm{BL} / 6$ mice was measured using a fluorescence spectrophotometer (FlourMax-4, HORIBA, Tokyo, Japan). The mitochondrial membrane potential in MIN-6 cells was measured using a cell sorter (BD FACSAria II, BD Biosciences, Sandra Jose, CA, USA).

\subsection{Protein extracts and western blot analysis}

Protein was extracted from the mouse pancreas and MIN-6 cells using RIPA lysis buffer (P0013B), which was purchased from Beyotime Institute of Biotechnology (Shanghai, China). The lysis buffer mainly contained $50 \mathrm{mM}$ of Tris (pH 7.4), $150 \mathrm{mM} \mathrm{NaCl}$, 1\% Triton $\mathrm{X}-100,1 \%$ sodium deoxycholate, $0.1 \%$ SDS and
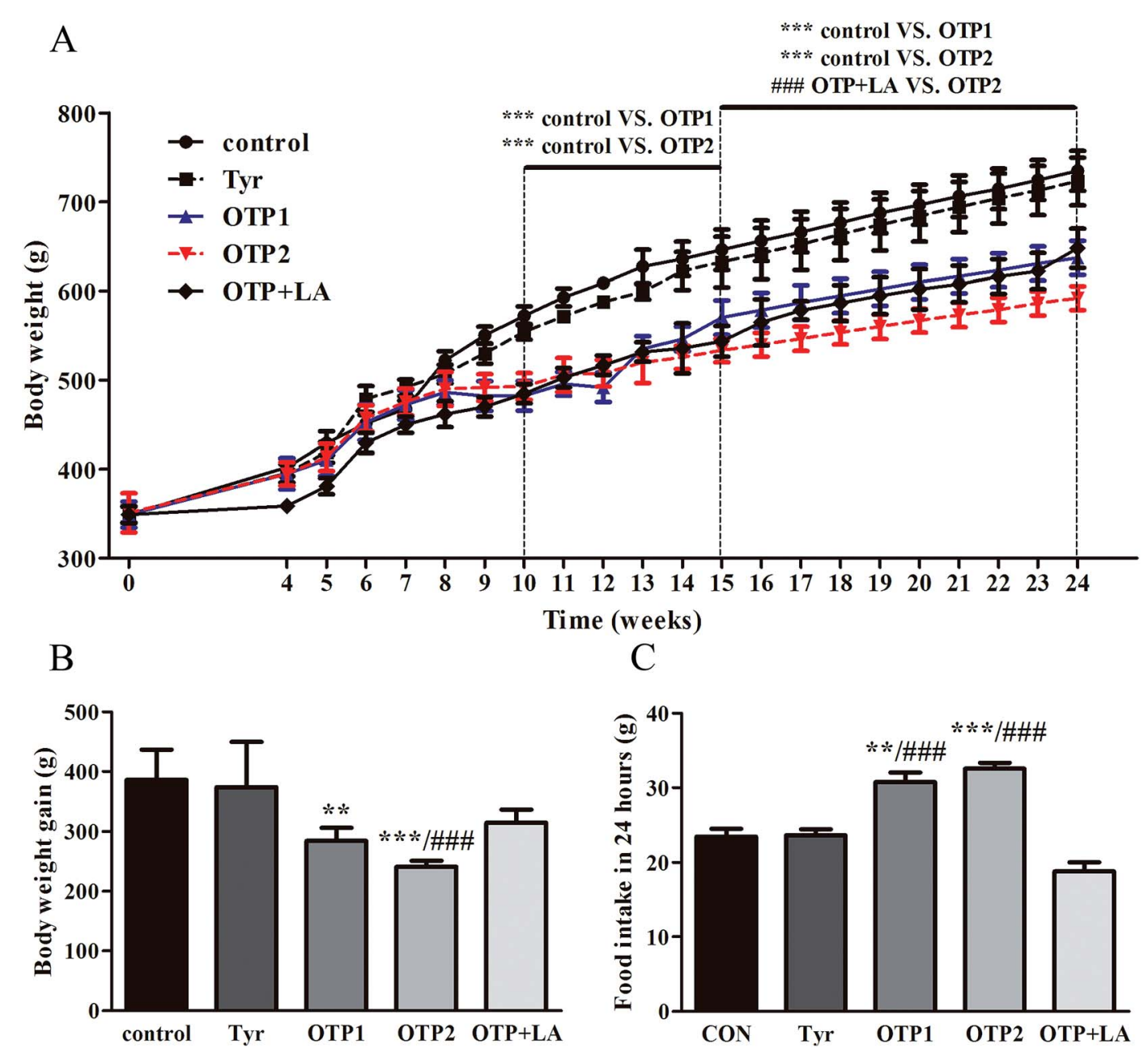

Fig. 1 Effects of OTPs on body weight and daily food intake in a 24 week dietary OTPs experiment. (A) OTPs affected the body weight growth of rats. The curves show the time-dependent body weight growth. (B) The bars show the body weight gain after the 24 week experimental period.

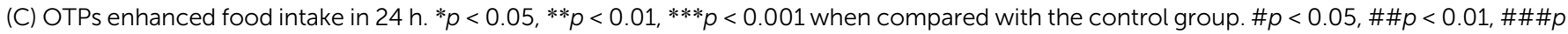
$<0.001$ when compared with the OTP + LA group. 
protease inhibitors, including sodium orthovanadate, sodium fluoride, EDTA and leupeptin. According to the manufacturer's instructions, $1 \mathrm{mM}$ (final concentration) phenylmethanesulfonyl fluoride (PMSF) was added to the lysis buffer before use. $200 \mathrm{mg}$ of pancreas and cells were homogenized in $1.5 \mathrm{~mL}$ lysis buffer on ice. After complete lysis, the homogenate was centrifuged for $5 \mathrm{~min}$ at $12000 \mathrm{~g}$. Protein concentration in the supernatant was determined by the BCA assay kit from Nanjing Jiancheng Bioengineering Institute (Nanjing, China), according to the instructions of the manufacturer. All extracts were mixed with $5 \times$ loading buffer $\left(50 \mathrm{mmol} \mathrm{L}{ }^{-1} \mathrm{pH}^{-1} 6.8\right.$ Tris-HCl, $100 \mathrm{~mm} \mathrm{~L}^{-1}$ dithiothreitol, $2 \%$ SDS, $0.1 \%$ bromophenol blue, $10 \%$ glycerol and $5 \% \beta$-mercaptoethanol) in the ratio of $4: 1$, and were then boiled at $95{ }^{\circ} \mathrm{C}$ for $10 \mathrm{~min}$ to denature the protein.

Protein extracts were separated on $12 \%$ SDS-PAGE gel using $10 \mu \mathrm{g}$ protein per sample and then transferred onto the NC membrane. The membrane was blocked in Tris-buffered saline (pH 7.4) with 5\% BSA and $0.1 \%$ Tween 20. Afterwards, the blocked membrane was incubated with primary antibody, including rabbit MT-ND1 antibody (ab74257, Abcam, Shanghai, China) and rabbit $\beta$-actin antibody (ab8226, Abcam, Shanghai, China) at a dilution of $1: 1000$ at $4{ }^{\circ} \mathrm{C}$ for 10 hours. Blots were incubated with secondary antibody (Sigma-Aldrich, U.S.A.) at a dilution of $1: 4000$, and fluorescence images were obtained with the automatic chemiluminescence imaging analysis system (Tannon 5200, Shanghai, China).

\subsection{Total mRNA isolation and quantitative RT-PCR (qRT- PCR)}

Total RNA of cells and tissues was extracted with Trizol reagent according to the manufacturer's protocol (Applied Biosystems, Foster City, CA, USA). The concentration of total RNA in each

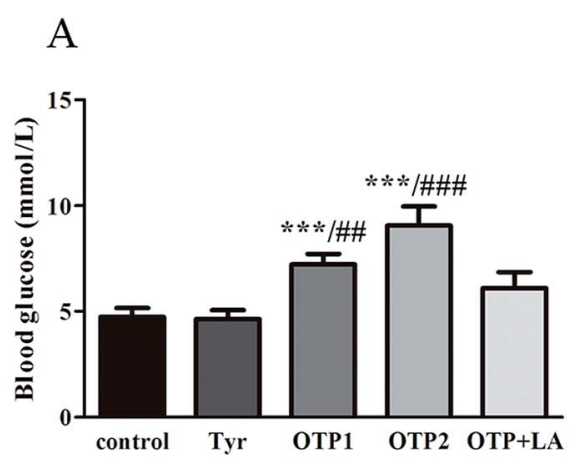

$\mathrm{C}$

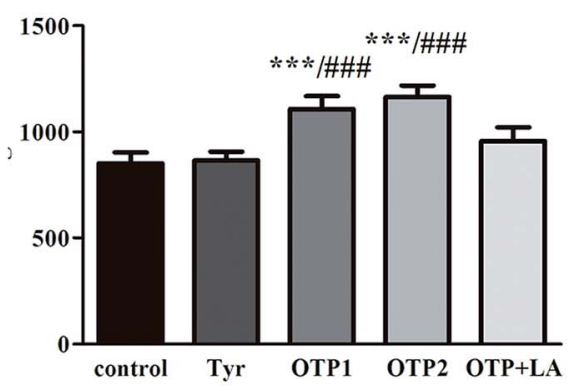

B

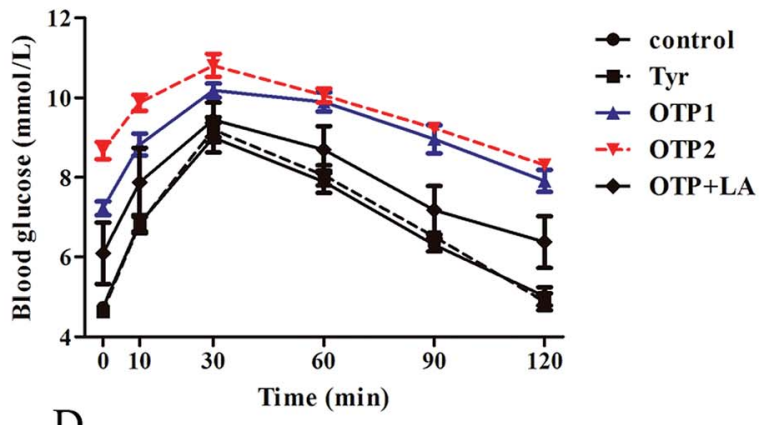

$\mathrm{D}$

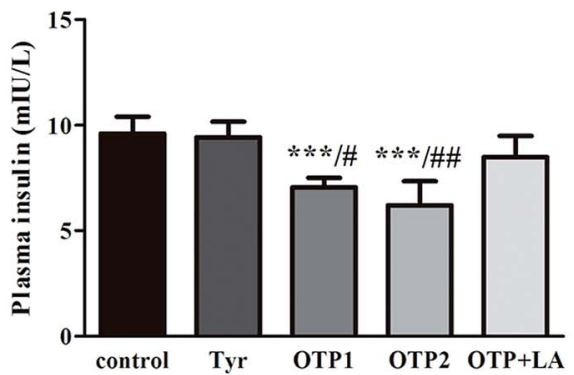

$\mathrm{E}$

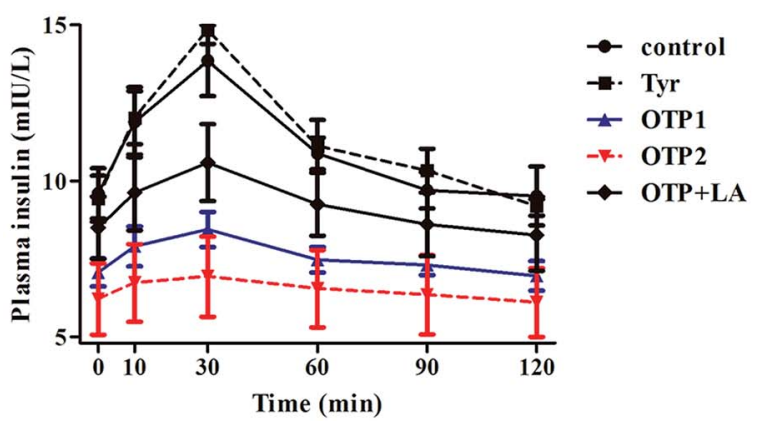

Fig. 2 Effects of OTPs on glucose tolerance and insulin synthesis in the 24 week dietary OTPs experiment. (A) OTPs elevated fasting blood glucose levels. (B) Blood glucose curve in GTT. (C) Area under the blood glucose curve in GTT. (D) OTPs decreased fasting plasma insulin levels.

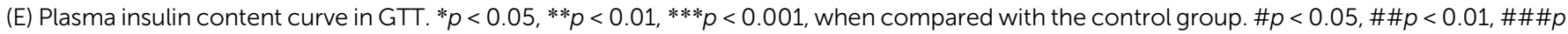
$<0.001$ when compared with the OTP + LA group. 
sample was quantified by NanoDrop Spectrophotometer (ND2000, Thermo, Waltham, MA, USA). A SYBR green based qRT-PCR kit was used according to the manufacturer's instructions in a $7900 \mathrm{HT}$ instrument (Applied Biosystems, Forster, CA, USA). The specificity of the product was assessed from melting curve analysis. Gene expressions were determined using the $2^{-\Delta \Delta C_{\mathrm{t}}}$ method. The primer's sequences for the genes are shown in Tables 1 and 2 .

\subsection{Statistical analysis}

All measurement values were expressed as mean \pm S.E.M. Significant differences between groups were determined by oneway analysis of variance using SPSS 20.0 software (SPSS Inc. Chicago IL., U.S.A.) for Windows. A difference was considered significant at the $p<0.05$ level.

\section{Results}

\subsection{4 week dietary OTP experiment in SD rats}

3.1.1. Effects of OTP on body weight, food intake, blood glucose, and insulin secretion. Fig. 1 shows the body weight and daily food intake. The body weight of the rats in the OTP groups became significantly lower than those of the control group after the 10th week of feeding. The body weight gains of the rats in the OTP groups were lower than those of the rats in the control group. The daily food intake was significantly higher in the
OTPs-treated rats than in the control group. LA prevented the body weight loss and appetite increase induced by OTPs.

Dramatic changes in body weight and increased food intake are major risk factors for diabetes. The results of the glucose tolerance test are shown in Fig. 2. Blood glucose levels under fasting conditions ( $0 \mathrm{~min}$ ) were higher in the OTPs-fed rats, compared to the control rats. Quantitatively, the area under the glucose curve was significantly increased in the OTPs-fed rats, as compared to that in the control rats. The plasma insulin level under fasting conditions ( $0 \mathrm{~min}$ ) was decreased in the rats in the OTP groups. Thirty minutes after glucose administration, plasma insulin levels increased to the maximum value and decreased rapidly in the control rats. However, there was no time-dependent change in insulin content in the OTPs-fed rats.

Fig. 3 shows the expression of genes (Glut2 and Gck) and transcription factors ( $P d x-1$ and $M a f A)$ involved in insulin biosynthesis in the pancreas were significantly lower in OTPstreated rats when compared with rats in control group. LA mitigated the effect of OTPs on blood glucose and insulin synthesis. These data point to impaired glucose tolerance and reduced insulin secretion in the OTPs-fed rats.

3.1.2. Effects of OTPs on oxidative stress, lipid peroxidation and protein oxidation. The levels of oxidative stress biomarkers are shown in Fig. 4. From the statistical analysis of the ESR signal of each group, the level of ROS in blood was significantly higher in the OTPs-treated groups, which was accompanied
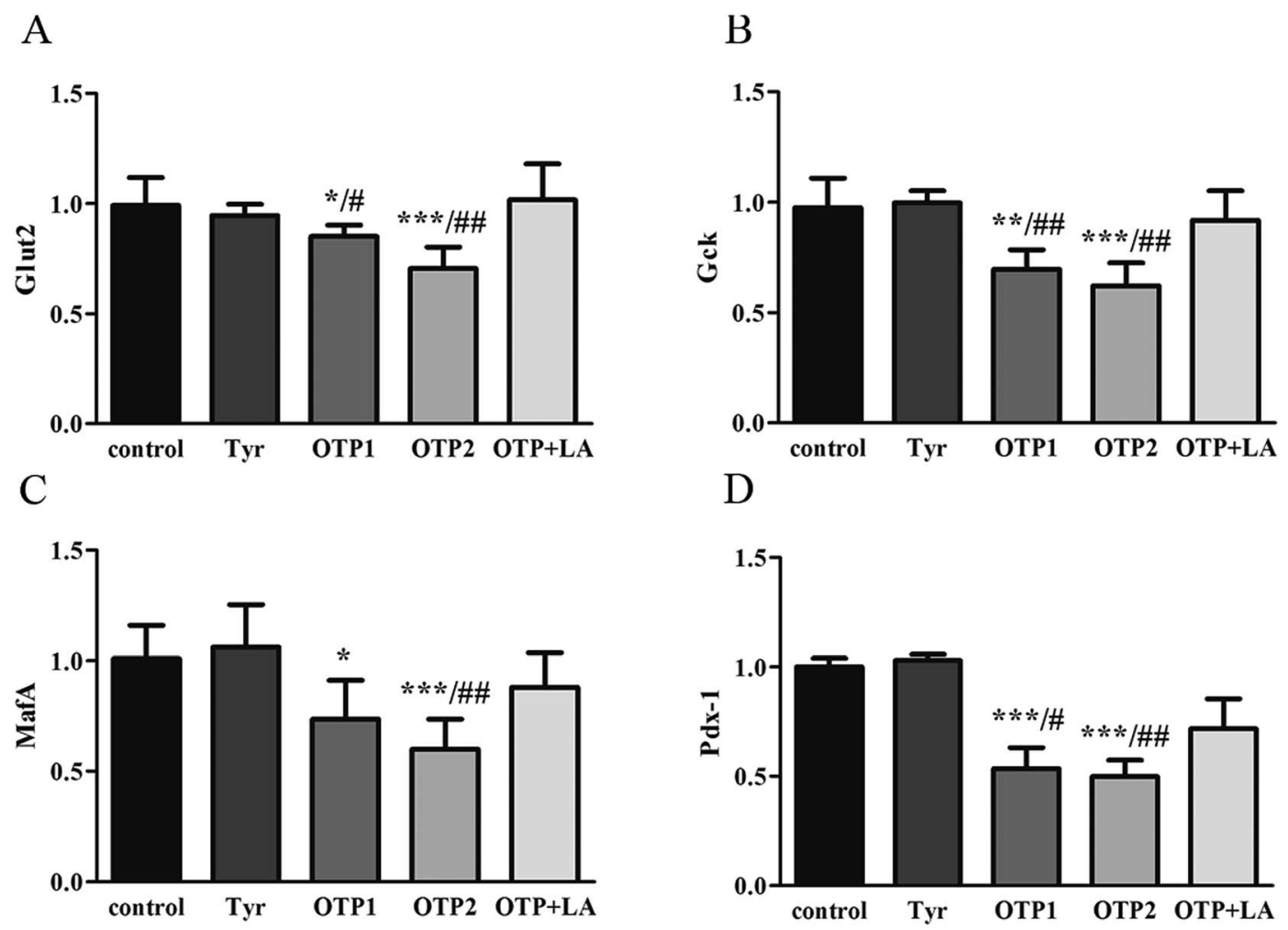

Fig. 3 Effects of OTPs on the mRNA of insulin synthesis related genes in the 24 week dietary OTPs experiment. (A) Glut 2 mRNA expression (relative to the control). (B) Gck mRNA expression (relative to the control). (C) MafA mRNA expression (relative to the control). (D) Pdx-1 mRNA

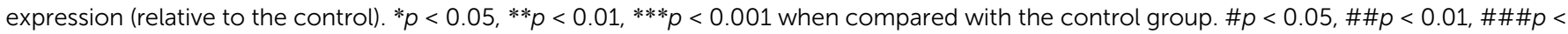
0.001 when compared with the OTP + LA group. 
A
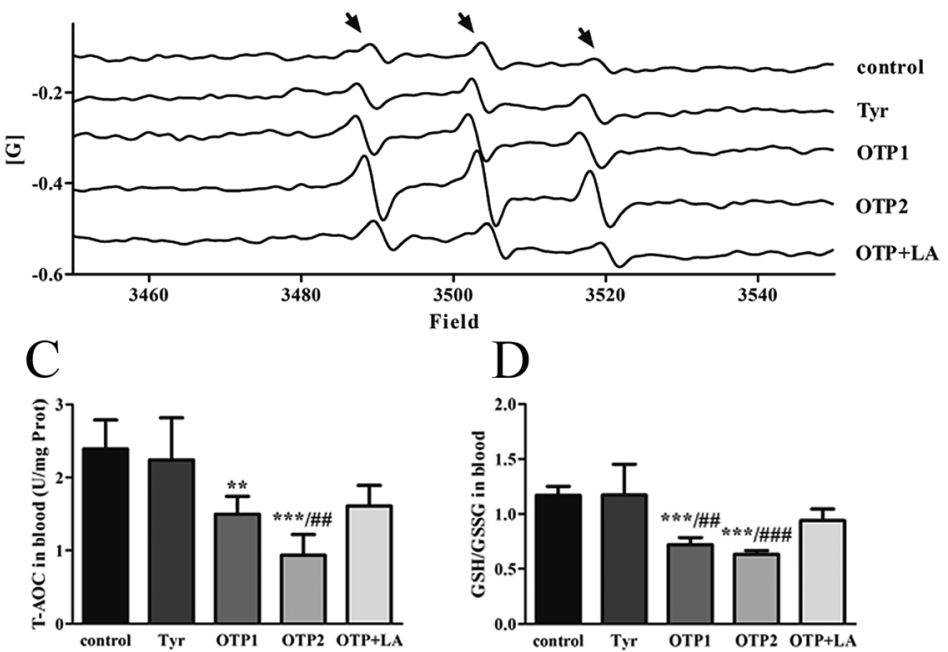

E

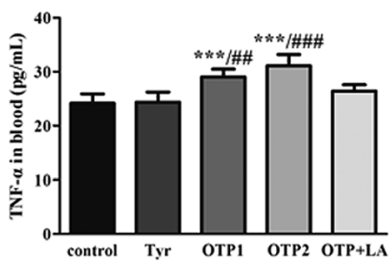

D

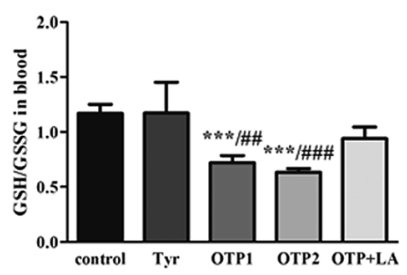

F

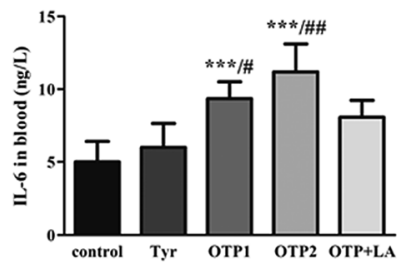

B

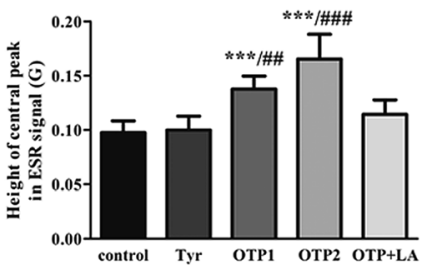

Fig. 4 Effects of OTPs on the redox status in blood in 24 week dietary OTPs experiment. (A) ESR signal of blood ROS. (B) OTPs induced excessive ROS production in blood. The bars show the height of the central peak in the ESR signal. (C and D) OTPs altered the T-AOC and GSH/GSSG ratios in blood. (E and F) OTPs enhanced the levels of IL- 6 and TNF- $\alpha$ in blood. $* p<0.05, * * p<0.01, * * * p<0.001$ when compared with the control group. $\# p<0.05, \# \# p<0.01, \# \# \# p<0.001$ when compared with the OTP + LA group.

by decreased total antioxidant capacity (T-AOC) and decreased the GSH/GSSG ratio in the blood. The levels of inflammatory markers (IL-6 and TNF- $\alpha$ ) were markedly higher in the blood of OTP-fed rats, compared to the controls. Fig. 5 shows that the antioxidant enzyme activities of the pancreas, including SOD, CAT, and GPx, were markedly lower in the OTPs-fed rats than in the controls. Simultaneously, the levels of MDA, Dityr, and 3-NT significantly increased in the pancreas of OTPs-fed rats. The OTPs treatment also downregulated the mRNA expression of antioxidant-related genes in the Nrf2/ARE pathway (Nrf2, Gpx-1, and Ho-1). Treatment with LA ameliorated the oxidative stress induced by OTPs. These data suggest that OTPs would induce oxidative stress and decrease the antioxidant capacity of the pancreas.

\subsection{0 week gavage experiment with OTPs and Dityr in C57BL/6 mice}

3.2.1. The effects of OTPs and Dityr on glucose tolerance and the redox status of $\mathrm{C57BL} / 6$ mice. As shown in Fig. 6. The gavage of OTPs and Dityr resulted in impaired glucose tolerance and decreased plasma insulin levels. OTPs and Dityr also downregulated the mRNA levels of genes related to the GSIS pathway (Fig. 7), induced excessive ROS generation in the blood, and decreased the T-AOC and GSH/GSSG ratio in the blood (Fig. 8). Dityr accumulated in the pancreas of the mice treated with OTPs or Dityr, suggesting that the pancreas was the target organ of
Dityr. Both OTPs and Dityr led to oxidative stress and decreased activities of antioxidant enzymes (Fig. 9). All the changes, including increased blood glucose levels, decreased insulin concentration, and impaired antioxidant capacity observed in the C57BL/6 mice after gavage were consistent with the effects induced by OTPs in the SD rats. There was no significant difference between the OTPs and Dityr treated mice. These data imply that Dityr is responsible for the pancreatic damage caused by OTP.

3.2.2. The effects of OTPs and Dityr on the mtDNA, mitochondrial membrane potential, and mRNA expression of genes involved in mitochondrial function and metabolism in the pancreas of the C57BL/6 mice. As shown in Fig. 10, the mtDNA/ nDNA ratio of the pancreas was significantly lower in the OTPS or Dityr treated mice than in the controls. The results of the western-blot assay showed that the OTPs or Dityr treatment induced a remarkable decrease in the protein level of a subunit of respiratory complex I (ND1). The ratios of JC-1 monomers and JC-1 polymers were markedly lower in the pancreas of the OTPs/Dityr treated mice, compared to those of the controls, indicating that OTPs/Dityr induced the mitochondrial membrane depolarization of the pancreas. In addition, the treatment of the mice with OTPs or Dityr decreased the mRNA levels of TFAM, ATP6, citrate synthase, OGDH, and it promoted the expression of UCP2. Furthermore, the OTPs/Dityr treatments dramatically suppressed the mRNA expression of the $\mathrm{K}_{\mathrm{ATP}}$ channel subunits Kir6.2 and Sur. 

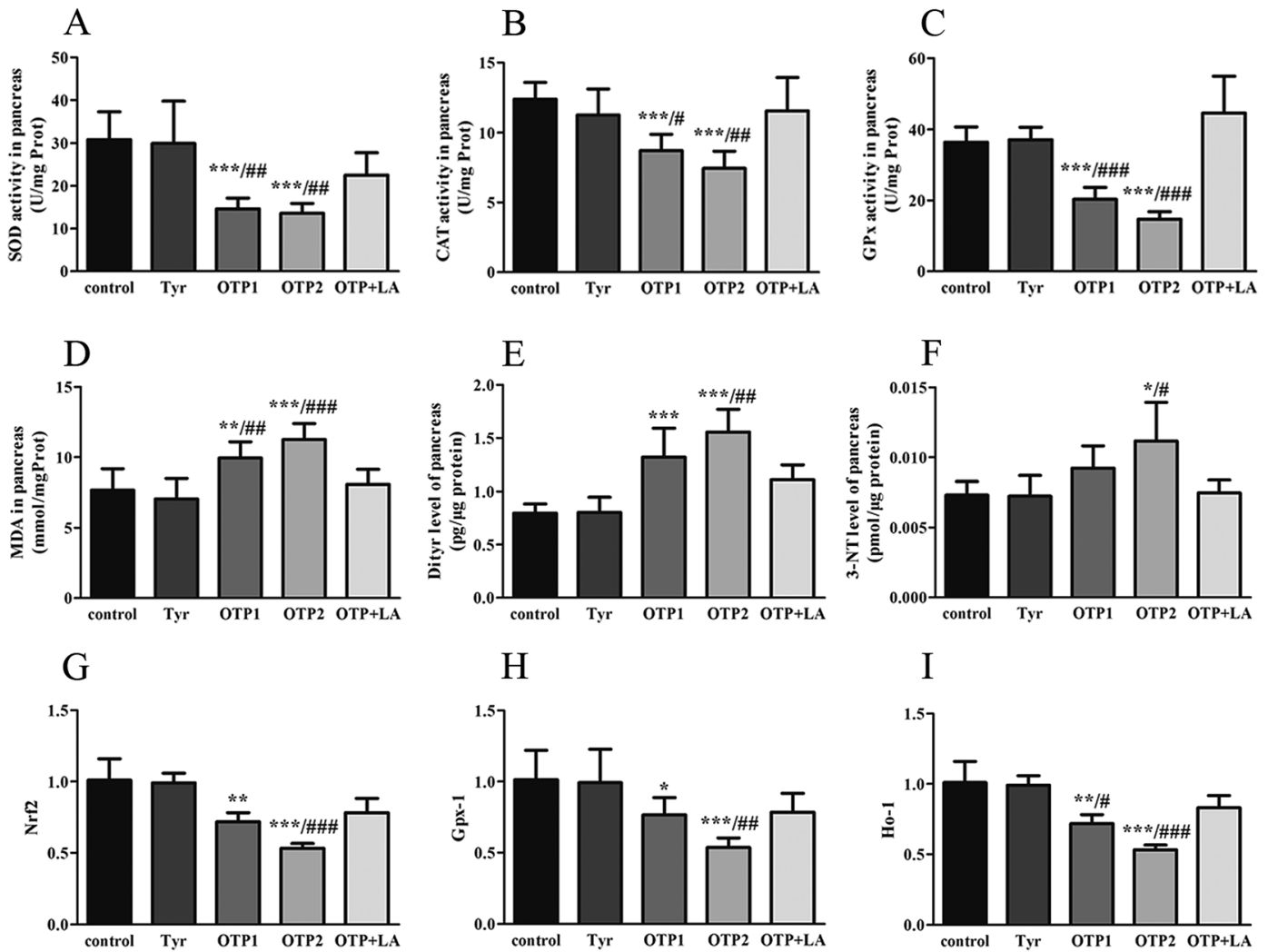

Fig. 5 Effects of OTPs on the redox status and antioxidant capacity of the pancreas in the 24 week dietary OTPs experiment. (A-C) OTPs inhibited the activities of antioxidant enzymes. (A) SOD; (B) CAT; (C) GPx. (D) OTPs enhanced the MDA level in the pancreas. (E) OTPs enhanced the Dityr levels in the pancreas. (F) OTPs enhanced the 3-NT level in the pancreas. (G-I) OTPs decreased the mRNA levels of genes in the Nrf2/ ARE pathway in pancreas. (G) Nrf2; (H) Gpx-1; (I) Ho-1. *p<0.05, **p<0.01,***p<0.001 when compared with the control group. \#p $<0.05$, \#\#p $<0.01, \# \#$ \# $<0.001$ when compared with the OTP + LA group.

A

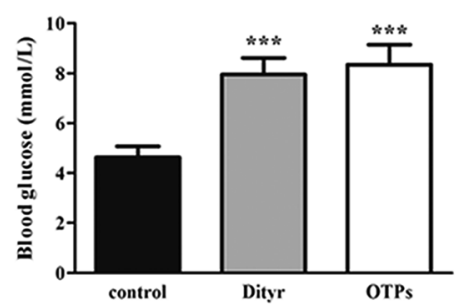

$\mathrm{D}$

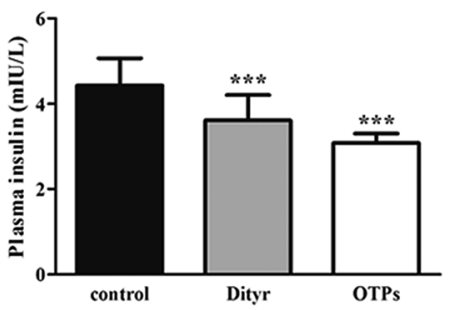

$\mathrm{B}$

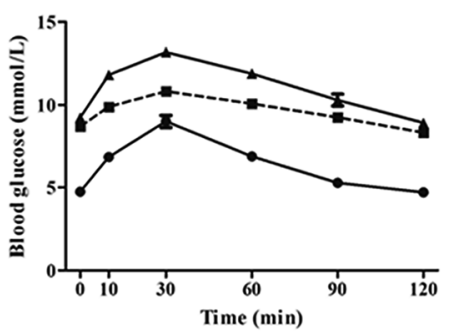

$\mathrm{E}$

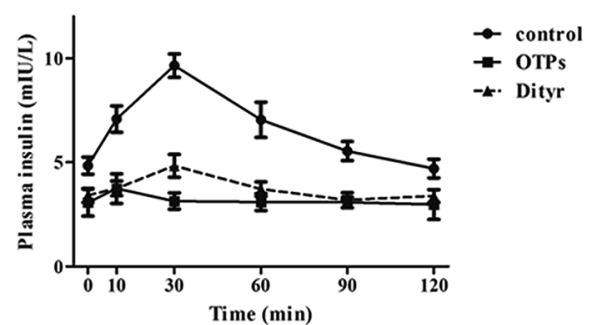

Fig. 6 Effects of OTPs/Dityr on glucose tolerance and insulin synthesis in the 10 week gavage experiment. (A) OTPs/Dityr elevated the fasting blood glucose level. (B) Blood glucose curve in GTT. (C) Area under the blood glucose curve in GTT. (D) OTPs/Dityr decreased the fasting plasma insulin level. (E) Plasma insulin content curve in GTT. *p<0.05, **p $<0.01, * * * p<0.001$. 

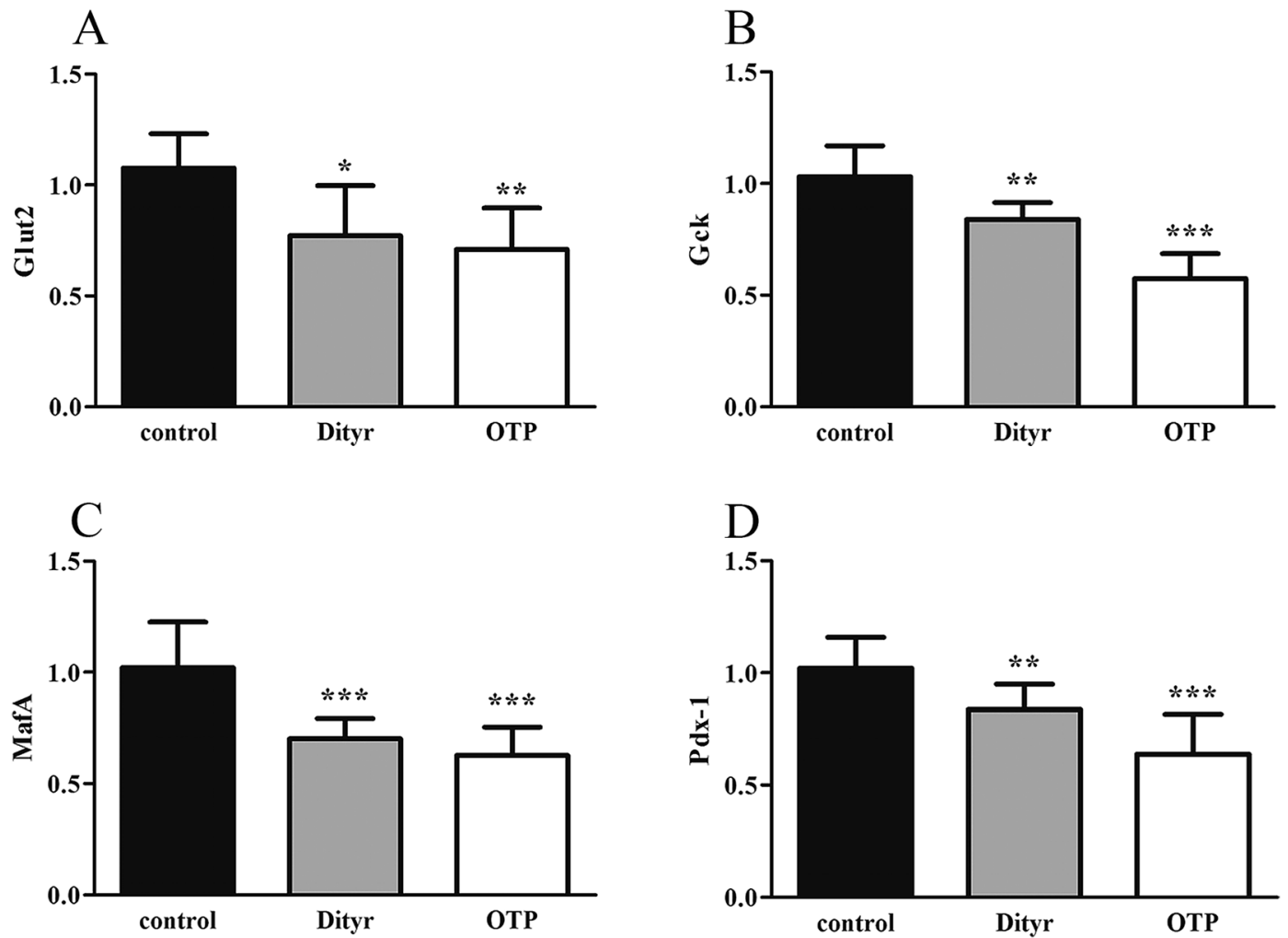

Fig. 7 Effects of OTPs/Dityr on the mRNA expression of insulin synthesis related genes of the pancreas in the 10 week gavage experiment. (A) Glut2. (B) Gck. (C) MafA. (D) Pdx-1. *p $<0.05, * * p<0.01, * * * p 0.001$.

A

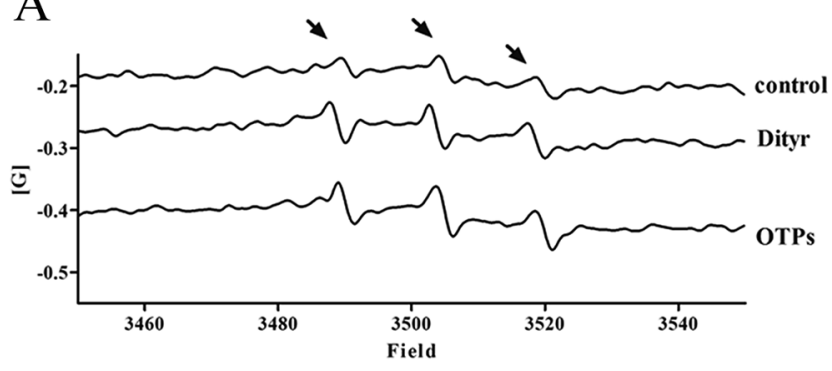

B

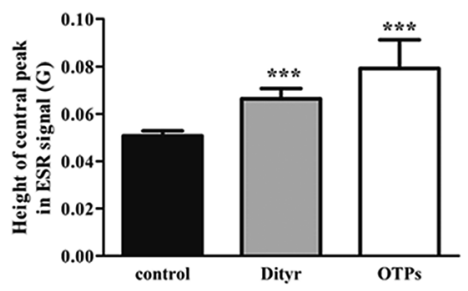

$\mathrm{C}$

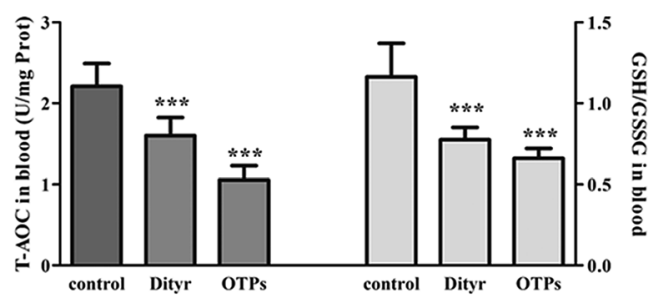

$\mathrm{D}$

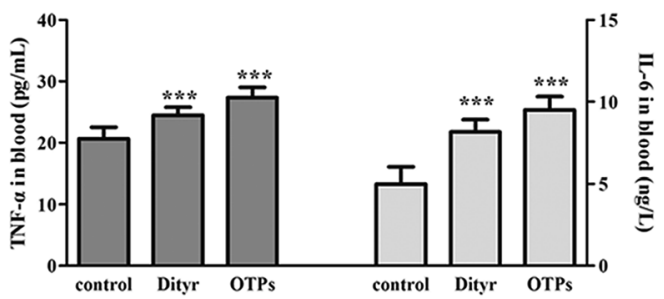

Fig. 8 Effects of OTPs/Dityr on the redox status of mice in the 10 week gavage experiment. (A) ESR signal of blood ROS. (B) OTPs/Dityr induced excessive ROS production in blood. The bars show the height of the central peak in the ESR signal. (C) OTPs/Dityr altered the T-AOC and GSH/ GSSG ratios in blood. (D) OTPs enhanced the levels of IL- 6 and TNF- $\alpha$ in blood. * $p<0.05, * * p<0.01, * * * p<0.001$.

3.2.3. The effects of OTP and Dityr on the histopathological changes and apoptosis in pancreatic islets. As shown in Fig. 11, at the morphological level, islets from the OTPs/Dityr-treated mice showed a disarray of cellular architecture and irregular boundaries. The percentage of TUNEL-positive areas was much larger in the pancreatic islets of the OTPs or Dityrtreated rats, suggesting that the OTPs or Dityr treatment caused apoptosis in the pancreatic islets. Both the OTPs and Dityr treatment down-regulated the mRNA level of the antiapoptosis gene (Bcl-2) and up-regulated the mRNA levels of 
A

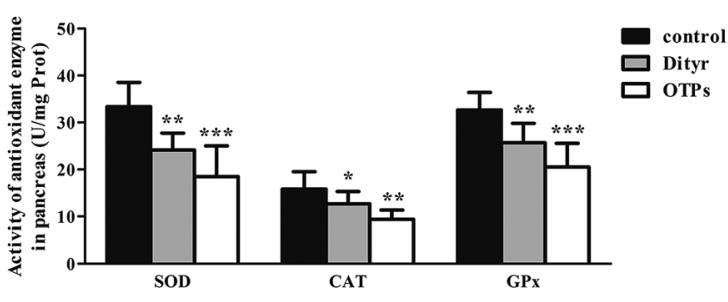

C

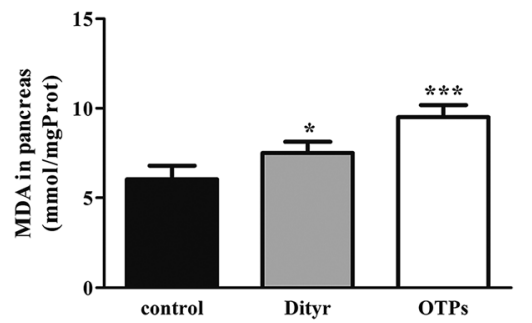

D

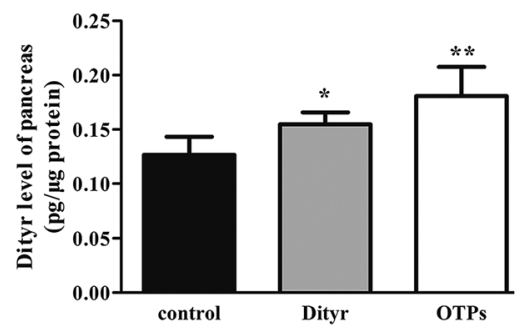

B

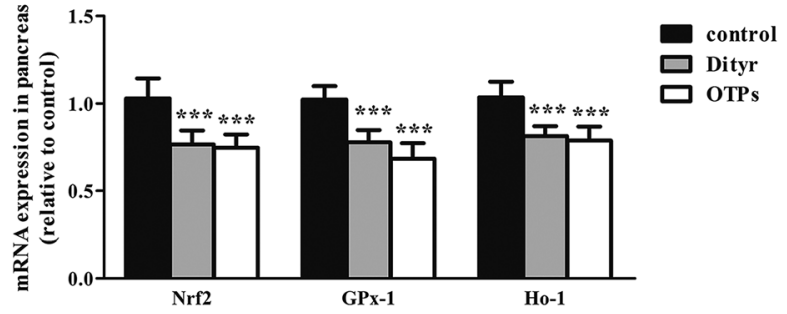

E

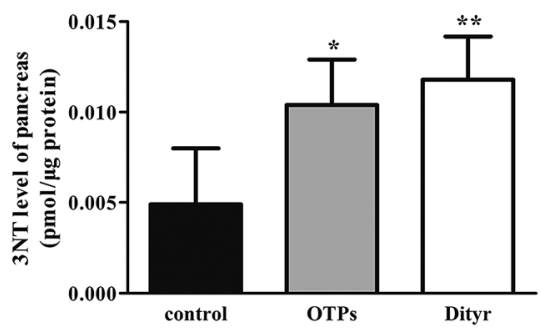

Fig. 9 Effects of OTPs/Dityr on the oxidative stress of mouse pancreas in the 10 week gavage experiment. (A) OTPs/Dityr inhibited the activities of antioxidant enzymes. (B) OTPs decreased the mRNA levels of genes in the Nrf2/ARE pathway in the pancreas. (C) OTPs/Dityr enhanced the MDA level in the pancreas. (D) OTPs/Dityr enhanced the Dityr levels in the pancreas. (E) OTPs/Dityr enhanced the 3-NT level in the pancreas. * $p<$ $0.05, * * p<0.01, * * * p<0.001$.

pro-apoptosis genes (Caspase 3, and Bax), indicating that OTPs and Dityr induced apoptosis of pancreatic cells by regulating apoptotic genes.

\subsection{Dityr exposure experiment using mice MIN-6 cells}

3.3.1. The effects of Dityr and $\mathrm{H}_{2} \mathrm{O}_{2}$ on the GSIS pathway and ROS generation in MIN-6 cells. Based on the above results, we found that Dityr and OTPs induced damage in the pancreas may be mainly related to oxidative stress. To clarify the mechanisms of Dityr induced oxidative stress, we performed the cell experiment using Dityr and $\mathrm{H}_{2} \mathrm{O}_{2}$. As shown in Fig. 12, Dityr affected basal insulin release (2.5 mM glucose) in MIN-6 cells, but the effect was not significant. However, Dityr resulted in a marked reduction in insulin secretion in cells when cultured in $16.7 \mathrm{mM}$ glucose. Furthermore, the ratio of GSIS to BIS was lower in the Dityr-treated cells. The mRNA levels of genes in the GSIS pathway were significantly decreased in the cells exposed to 0.1 and $1 \mu \mathrm{M}$ Dityr when compared with those of the untreated cells. Consistent with the effects of Dityr on the insulin synthesis in MIN-6 cells, $\mathrm{H}_{2} \mathrm{O}_{2}$ exposure also impaired the GSIS pathway in MIN-6 cells. We further found a significant increase in cellular ROS levels in both Dityr- and $\mathrm{H}_{2} \mathrm{O}_{2}$-treated cells. In brief, Dityr has a similar effect on oxidative stress as $\mathrm{H}_{2} \mathrm{O}_{2}$, which leads to an impaired GSIS pathway.

3.3.2. The effects of Dityr and $\mathrm{H}_{2} \mathrm{O}_{2}$ on mtDNA in MIN-6 cells. The mtDNA/nDNA ratio of the Dityr- $/ \mathrm{H}_{2} \mathrm{O}_{2}$-treated cells was significantly lower than that of the untreated cells, which suggested that exposure to Dityr or $\mathrm{H}_{2} \mathrm{O}_{2}$ led to a decline in the mtDNA copy number. The Dityr $/ \mathrm{H}_{2} \mathrm{O}_{2}$ treatment also induced a remarkable decrease in the protein level of ND1 in MIN-6 cells (Fig. 13).

3.3.3. The effects of Dityr and $\mathrm{H}_{2} \mathrm{O}_{2}$ on mitochondrial membrane potential, mRNA expression of genes involved in mitochondrial function and metabolism, and ATP generation in MIN-6 cells. After the MIN-6 cells were treated with 0.1 and 1 $\mu \mathrm{M}$ Dityr, a shift of fluorescence from red to green was observed, indicating that the Dityr induced mitochondrial membrane depolarization in the cells. The administration of Dityr also affected the mRNA expressions of genes involved in mitochondrial function and metabolism in MIN-6 cells. In addition, cellular ATP levels were reduced to $68.5 \%$ and $53.9 \%$ of that detected in the untreated cells after incubation with 0.1 and 1 $\mu \mathrm{M}$ Dityr, respectively. There was no difference in the ATP generation of the cells treated with $0.01 \mu \mathrm{M}$ Dityr and the unexposed cells. In line with these findings, the $\mathrm{H}_{2} \mathrm{O}_{2}$ exposure also led to mitochondrial membrane depolarization, decreased ATP generation, and altered mRNA expressions of genes involved in mitochondrial function and metabolism in MIN-6 cells (Fig. 14).

3.3.4. The effects of Dityr and $\mathrm{H}_{2} \mathrm{O}_{2}$ on apoptosis through the intrinsic mitochondrial pathway. Dityr reduced cell viability in a dose-dependent manner. Dityr up-regulated the expression of the pro-apoptotic gene (Bax) and down-regulated the expression of the anti-apoptotic gene Bcl-2. Caspase 3 expression markedly increased in the Dityr-exposed cells, compared to the untreated cells. $\mathrm{H}_{2} \mathrm{O}_{2}$ exposure also decreased cell viability and altered the mRNA expression of apoptosis related genes (Fig. 15). 
A

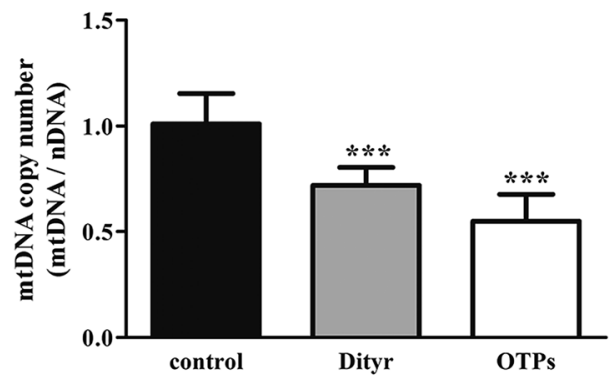

$\mathrm{B}$

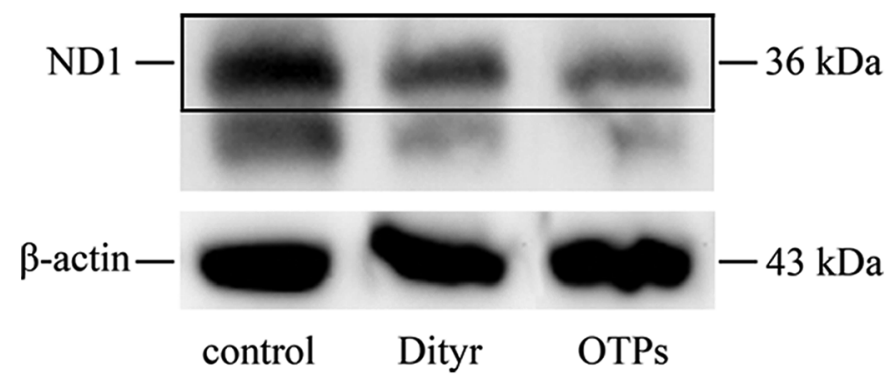

C

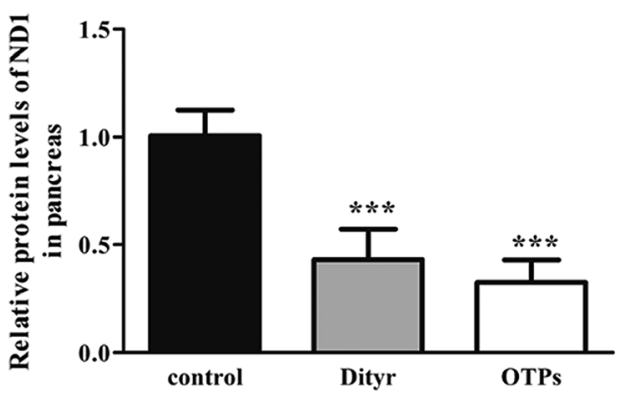

$\mathrm{D}$

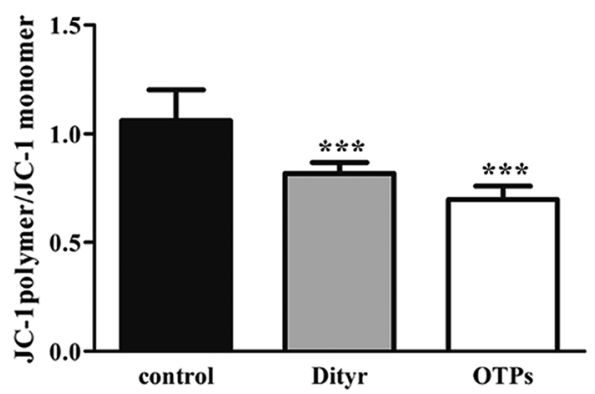

$\mathrm{E}$

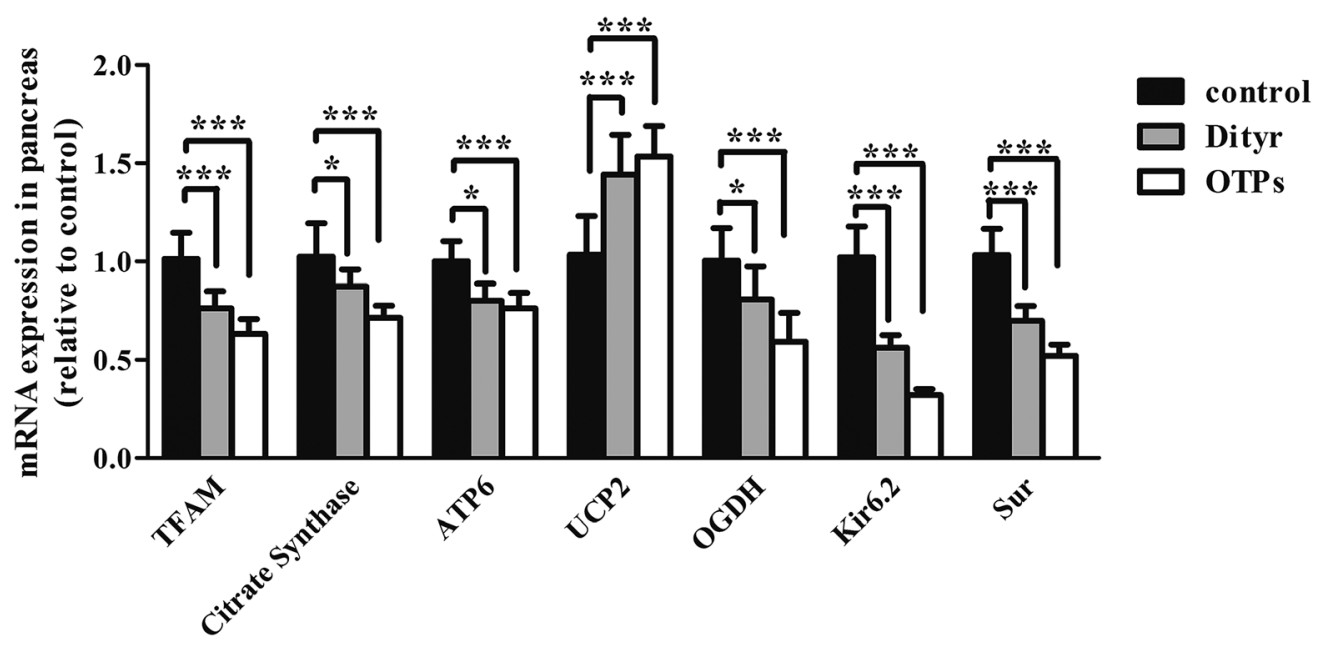

Fig. 10 Effects of OTPs/Dityr exposure on mtDNA and mitochondrial membrane potential of pancreas in 10-week gavage experiment. (A) OTPs/ Dityr declined the mtDNA copy number of pancreas. The bars show the ratio of mtDNA (ND2)/nDNA ( $\beta 2 M)$. (B and C) OTPs/Dityr declined the protein level of subunit of respiratory complex I: ND1 was measured by Western blot. $\beta$-Actin was used as an internal control. (D) Mitochondrial membrane potential measured by the JC-1 probe in MIN-6 cells. The bars show the JC-1 polymer/JC-1 monomer ratio. (E) OTPs/Dityr altered the mRNA expression of genes involved in mitochondrial metabolism and function. ${ }^{*} p<0.05 ; * * p<0.01 ; * * p<0.001$. 


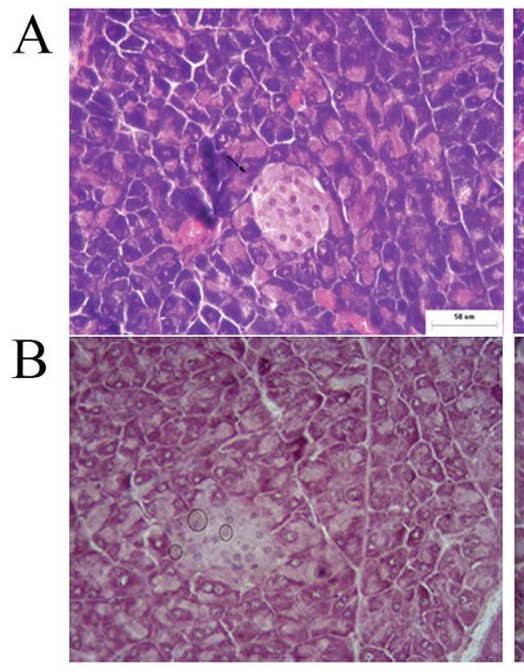

control

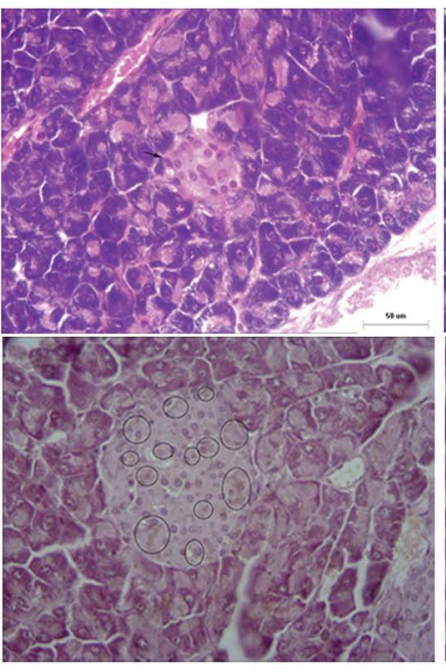

Dityr

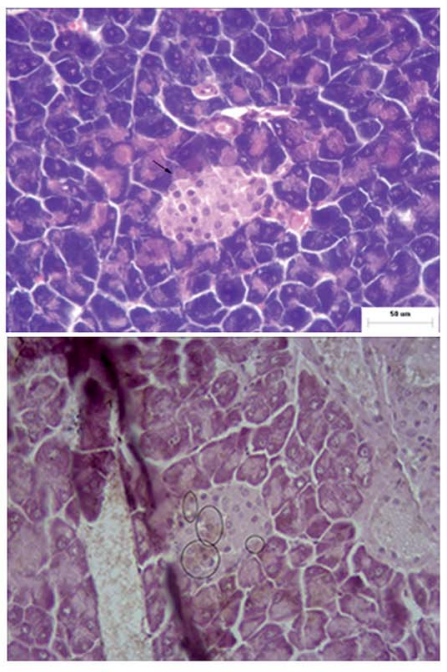

OTPs
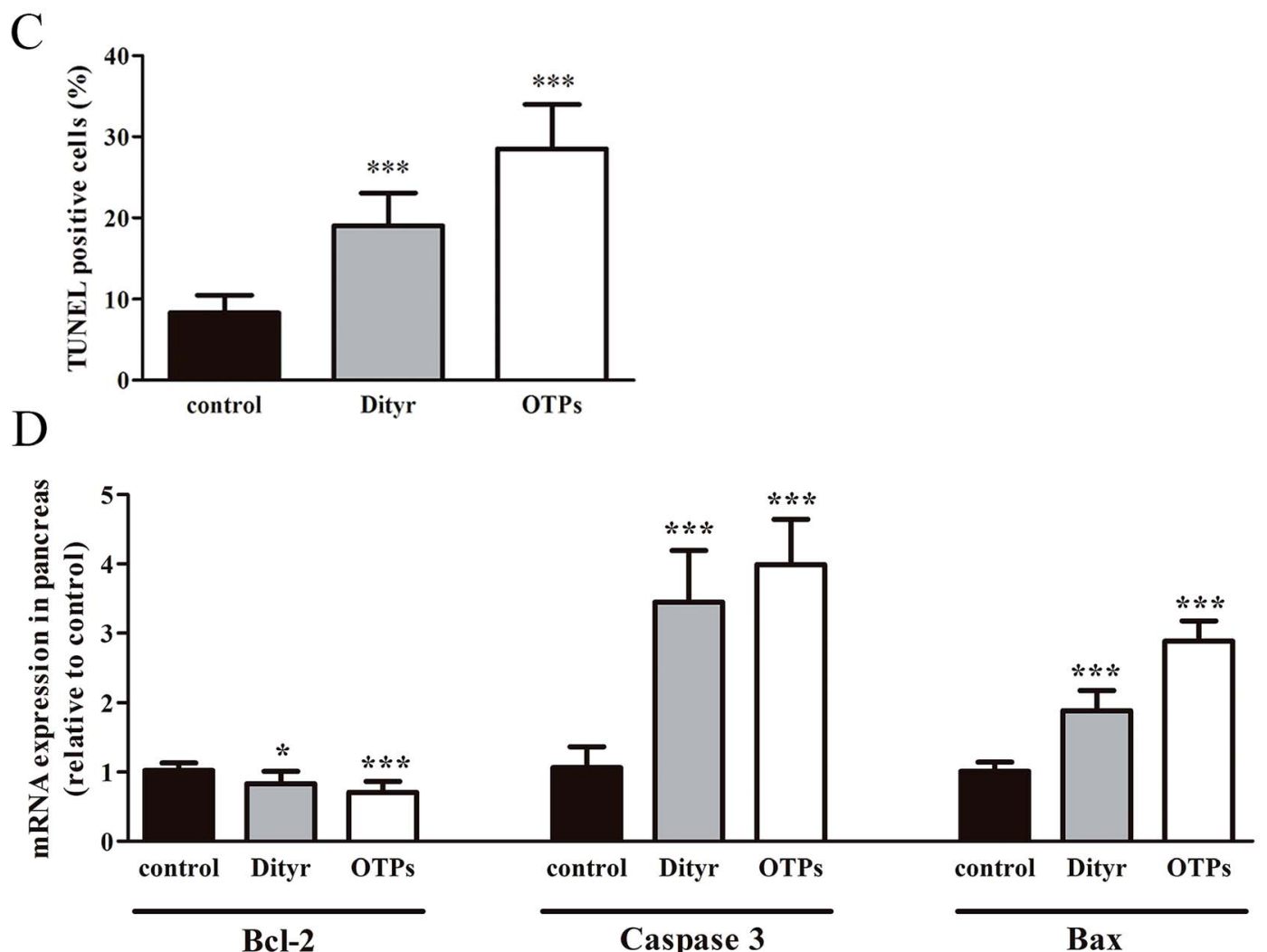

Fig. 11 Effects of OTPs/Dityr on histopathological changes and apoptosis of pancreatic islets (A) hematoxylin and eosin staining (arrows indicate $\beta$ cells mass, $400 \times$ ). (B) TUNEL staining of pancreas (cells in the circle are TUNEL-positive cells, $400 \times$ ). (C) TUNEL positive cells (\%). (D) Anti-/proapoptosis genes levels of pancreas. ${ }^{*} p<0.05, * * p<0.01, * * * p<0.001$.

\section{Discussion}

Tyrosine residues are susceptible to oxidation and nitration. The structures of oxidized tyrosine products (OTPs) are stable and not easily subjected to enzymatic hydrolysis; therefore, OTPs are widely detected in food systems, including meat and dairy products. ${ }^{\mathbf{3 0}, 31}$ We previously demonstrated that a diet high in OTPs resulted in oxidative injury and inflammation, ${ }^{32}$ which may be briefly associated with many metabolic disorders such as liver and renal fibrosis changes. The pancreas is particularly susceptible to oxidative stress, due to the weak activities of antioxidant enzymes, ${ }^{33}$ and it plays an important role in glucose metabolism; therefore, the pancreas may be attacked by the oxidative stress induced by OTPs. However, the impact of dietary OTPs on the pancreas is not clear. 
A

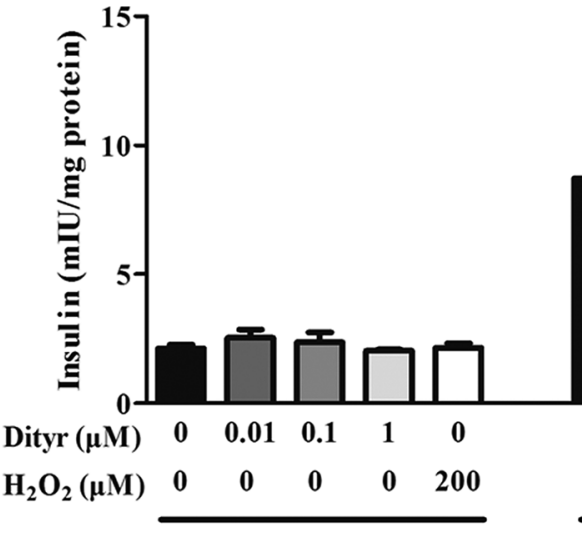

$2.5 \mathrm{mM}$ glucose

C

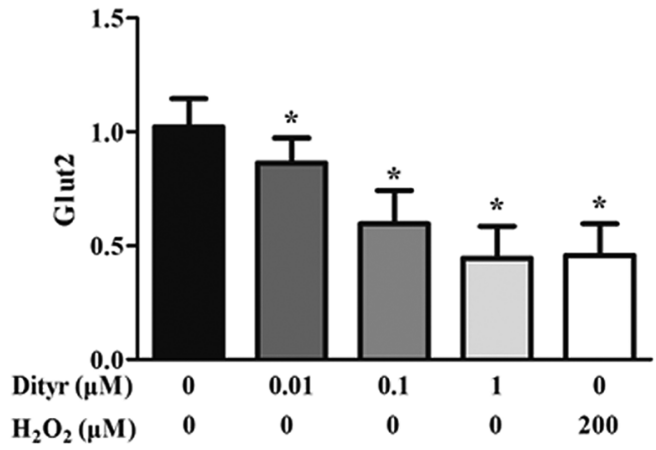

B

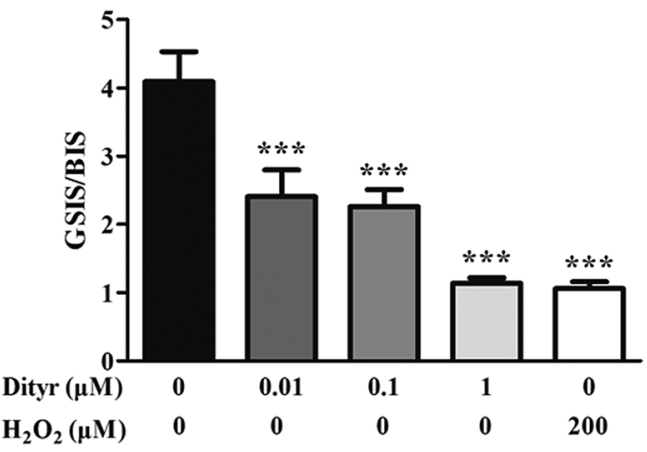

\section{$16.7 \mathrm{mM}$ glucose}

$\mathrm{D}$

E
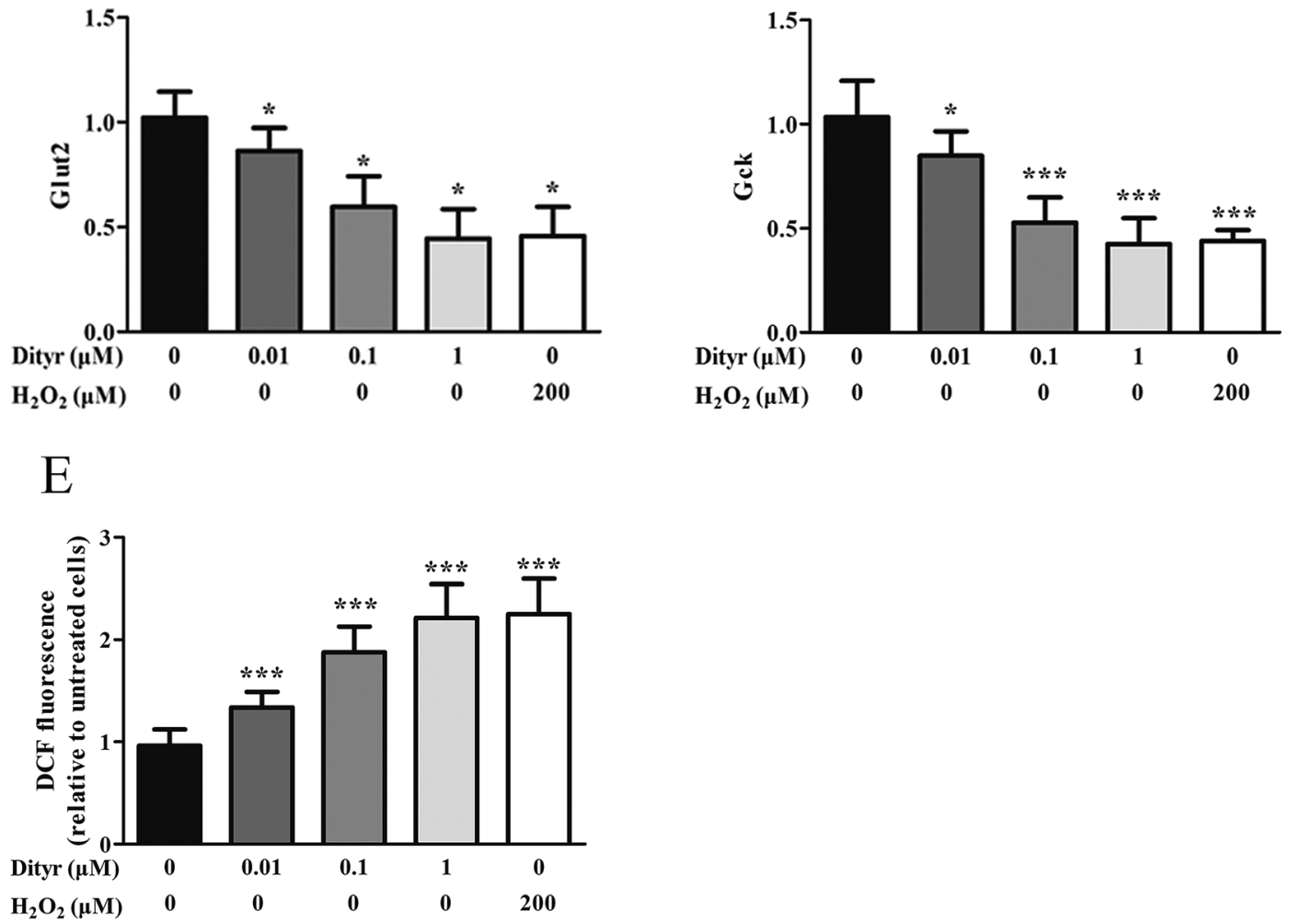

Fig. 12 Effects of Dityr on the glucose-stimulated insulin secretion (GSIS) pathway and ROS content in MIN-6 cells. (A and B) Dityr decreased the insulin secretion triggered by glucose. The levels of insulin were normalized to the protein content. ( $C$ and $D)$ Exposure to Dityr altered the mRNA expression of GSIS-related genes in MIN-6 cells. (C) Glut2; (D) Gck. (E) Dityr exposure induced excessive ROS generation in MIN-6 cells. * $p<$ $0.05 ; * *<0.01 ; * * p<0.001$.

The elevated fasting blood glucose and impaired glucose tolerance found in this study suggest that OTPs consumption would lead to dysfunction of the pancreatic islets. The OTPs treatment apparently resulted in down-regulation of mRNA expressions of genes in the GSIS pathway (Glut2 and Gck), as well as down-regulation of transcription factors that bind to promoters of insulin genes ( $P d x-1$ and MafA). These findings confirmed that the consumption of OTPs impaired the GSIS pathway via the regulation of glucose uptake and glycolysis. Past research on the redox imbalance in diabetes reported that exposure of $\beta$ cells to ROS suppressed insulin gene promoter activity and mRNA expression. ${ }^{34}$ In addition, ROS markedly reduced the binding of $\beta$ cells-specific transcription factors (Pdx-1 and MafA) to the insulin gene promoter ${ }^{35}$ In the present study, with increasing doses of OTPs, the generation of ROS and inhibition of antioxidant enzymes (SOD, CAT, and GPx) increased in rats, reflecting severe oxidative stress. It has been reported that glutathione plays an important protective role in 
A

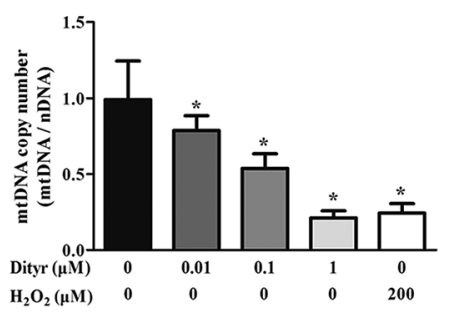

B
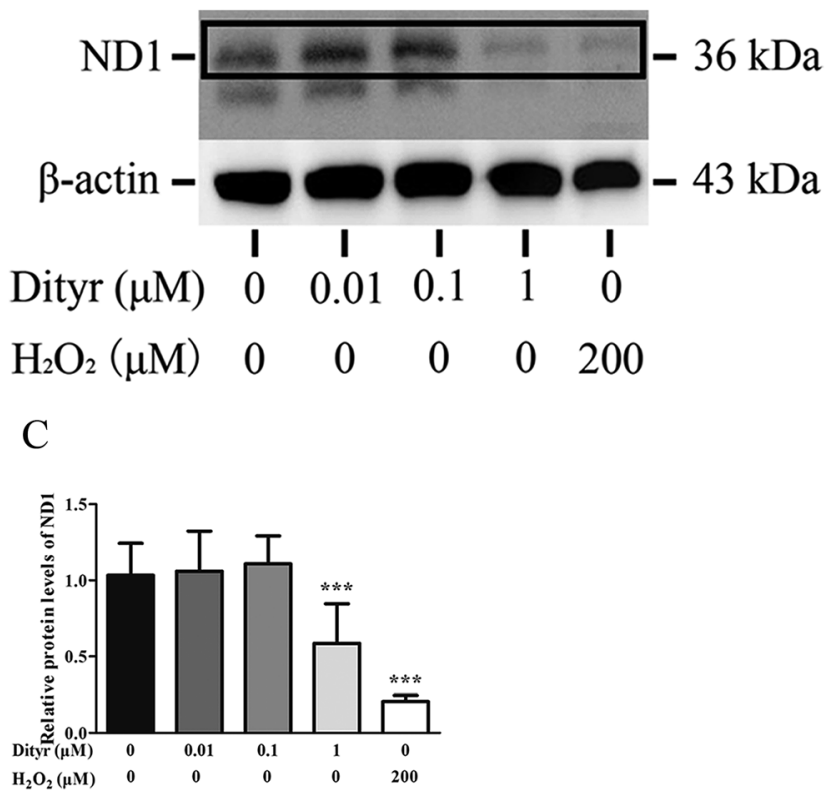

Fig. 13 Effects of Dityr on mtDNA in MIN-6 cells. (A) Dityr decreased the mtDNA copy number in MIN-6 cells. The bars show the ratio of $m t D N A(N D 2) / n D N A(\beta 2 M)$. (B and C) Dityr decreased the protein level of the subunit of respiratory complex I: ND1 was measured by western blot. $\beta$-Actin was used as an internal control. ${ }^{*} p<0.05$; $* p<0.01$; $* * * p<0.001$

combating the oxidative damage of biological molecules, and the ratio of GSH to GSSG often changes in the presence of oxidative stress. ${ }^{36}$ In the current study, we measured the levels of GSH and GSSG, and the results revealed a decline in the GSH level and a rise in the GSSG level. The GSH/GSSG ratio in the OTPs-fed rats decreased when compared to the controls. Excessive ROS generation plays a key role in chronic inflammation, ultimately resulting in increased levels of inflammatory cytokines, such as TNF- $\alpha$, IL- $6{ }^{37}$ Many previous studies revealed the co-effects of oxidative stress and chronic inflammation on the bio-function of the pancreas. ${ }^{38,39}$ In the present study, the co-effects of oxidative stress and increased expression of inflammatory cytokines could have led to the dysfunction of the pancreatic islets.

Oxidative damage is a complex pathophysiological process, which is implicated in a series of cellular and molecular events and signaling pathways. Among these, the Nrf2/ARE pathway has been identified as a potentially important pathogenesis mechanism in diabetes mellitus. ${ }^{40}$ The antioxidant response element (ARE) is located in the promoter region of some genes encoding antioxidant enzymes, including Gpx, glutathione $S$ transferases (Gst), heme oxygenase-1 (Ho-1), and $\gamma$-glutamylcysteine synthetase. ${ }^{41}$ Under normal conditions, Nrf2 binds to the Kelch-like ECH-associated protein-1 (Keap1) in the cytoplasm, in association with the actin cytoskeleton. In the presence of stress induced by electrophilic groups, Nrf2 dissociates from Keap1 and translocates to the nucleus. It then binds to ARE sequences, resulting in the transcriptional activation of antioxidant and phase II detoxifying genes. ${ }^{42} \mathrm{Nrf2}$ is activated under conditions of mild oxidative stress and inhibited under conditions of severe oxidative stress. ${ }^{43}$ In this study, the downregulation of $N r f 2$ expression might have contributed to excessive ROS generation. The findings of the down-regulated $N r f 2$, Gpx1, and Ho-1 expression suggest that the OTP treatment induced oxidative stress in the pancreas through inhibition of the Nrf2/ARE pathway. In the present study, L-tyrosine was used as a source to prepare OTPs. As the OTPs contained some tyrosine residues, a tyrosine-only group was used as a negative control. The rats in the tyrosine-treated group exhibited no oxidative stress or abnormal glucose metabolism, as compared to the control group. All these findings confirmed that the oxidative damage that occurred after the 24 week OTP treatment was specifically induced by the treatment.

Many antioxidants, such as lipoic acid (LA), have been reported to prevent or at least attenuate the damage caused by oxidative stress. ${ }^{44}$ Numerous studies have demonstrated the therapeutic potential of LA in diabetes, reporting that LA provided protection against oxidative damage and reduced the levels of inflammatory cytokines. ${ }^{45-47}$ In this study, the rats fed the OTP diet supplemented with LA had normal blood glucose and insulin levels. LA significantly inhibited OTP-induced oxidative damage via down-regulation of oxidative stress and up-regulation of the activities of antioxidant enzymes. In addition, LA significantly increased the expression of Nrf2, Gpx-1 and $H_{0-1}$ in the pancreas of OTPs-treated rats. These findings strengthen our hypothesis that a diet rich in OTPs would affect insulin secretion in rats, associated with impaired antioxidant capacity. We also suggest that antioxidant supplements may be a feasible therapeutic response to oxidative stress induced by protein oxidation.

Dityr is the main component of OTPs, which is a very unusual molecule, linked not by peptide bonds but by covalent bonds. These bonds are resistant to proteolytic degradation and acid hydrolysis. ${ }^{48}$ Therefore, we assumed that Dityr might be responsible for OTPs-induced injury and we further performed a 10 week gavage to study the effect of Dityr. The gavage study showed that OTPs and pure Dityr led to similar oxidative injuries in islets. Furthermore, the oxidative injury in the OTPs/ Dityr treated mice was consistent with that observed in the OTPs-fed rats. These results indicate that the administration of dietary OTPs induces oxidative stress and inflammation in the pancreas, thereby potentially promoting dysfunction of insulin synthesis in islets. The present study proved that Dityr, the main component of the OTPs mixture, has an important role in OTPsinduced oxidative stress. 
A
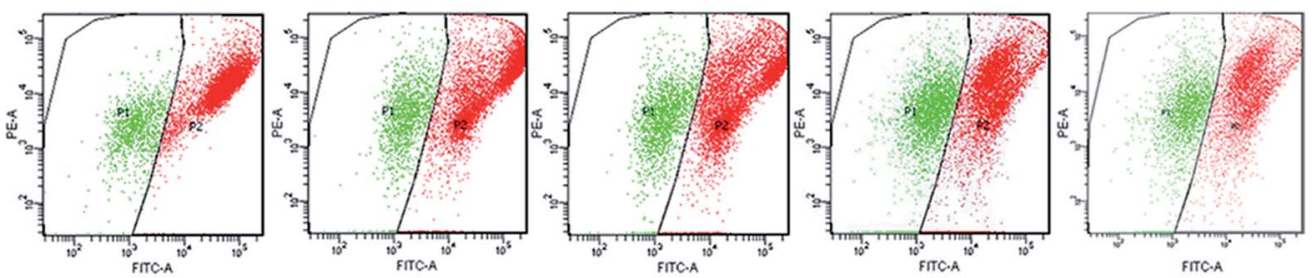

$\begin{array}{ll}\text { Dityr }(\mu \mathrm{M}) & 0 \\ \mathrm{H}_{2} \mathrm{O}_{2}(\mu \mathrm{M}) & 0\end{array}$

0.01

0

0.1

0

C
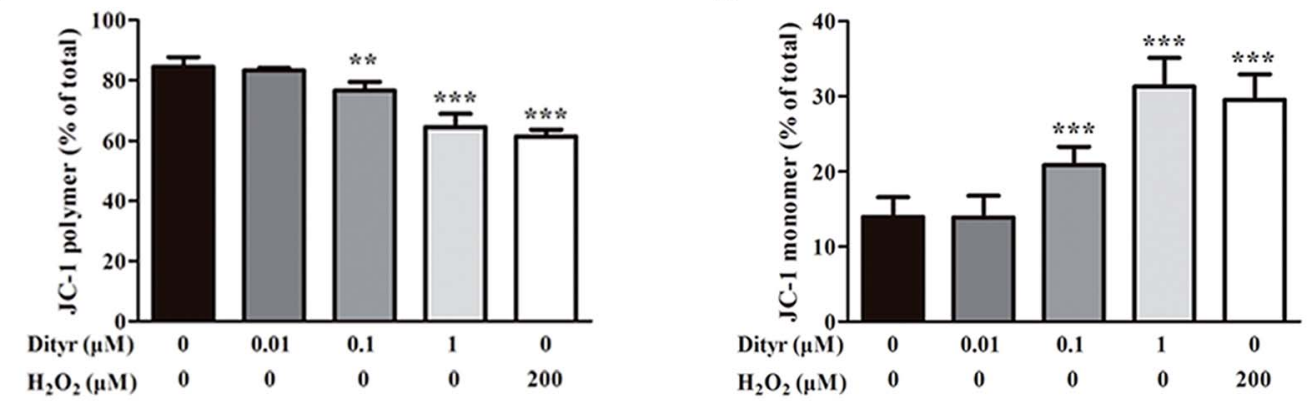

$\mathrm{D}$

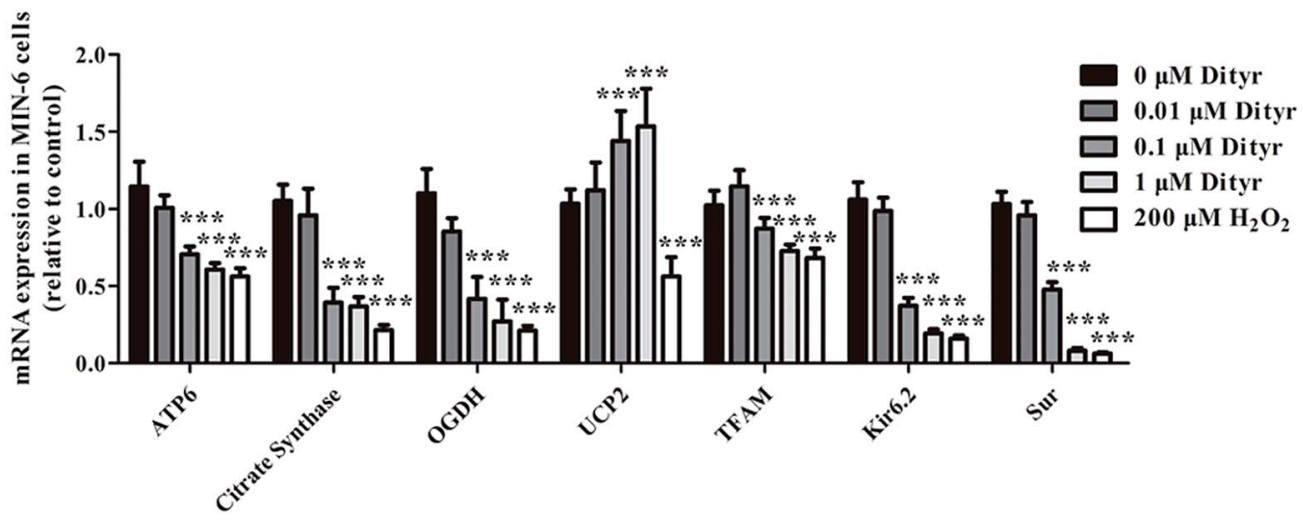

E

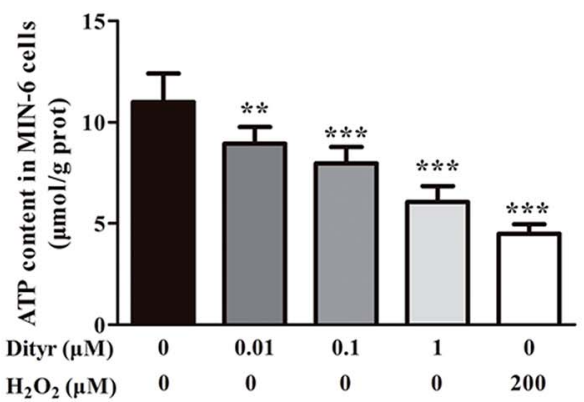

Fig. 14 Effects of Dityr on mitochondrial membrane potential mRNA expression of genes involved in mitochondrial metabolism and function, and ATP generation in MIN- 6 cells. (A) Mitochondrial $\Delta \psi_{m}$ measured by the JC-1 probe in MIN- 6 cells. (B and C) The bars show the percentage of JC-1 polymer-positive and JC-1 monomer-positive cells. (D) Dityr altered the mRNA expression of genes involved in the mitochondrial metabolism and function. (E) Dityr decreased the ATP generation in MIN-6 cells. ${ }^{*} p<0.05 ; * * p<0.01 ; * * p<0.001$.

The mitochondrion plays an important role in energy metabolism. ${ }^{49}$ It is well established that the mitochondrial oxidative phosphorylation system generates free radicals and that the electron transport chain is vulnerable to ROS, with excessive generation of ROS leading to mitochondrial dysfunction. ${ }^{50}$
Mitochondrial defects in $\beta$ cells disturb glucose-stimulated insulin synthesis (GSIS). ${ }^{19,25}$ In pancreatic $\beta$ cells, glucose transporter 2 (Glut2), which is phosphorylated by glucokinase (Gck), is involved in glucose transportation and glycolysis. In mitochondria, pyruvate enters into the tricarboxylic acid (TCA) cycle and 

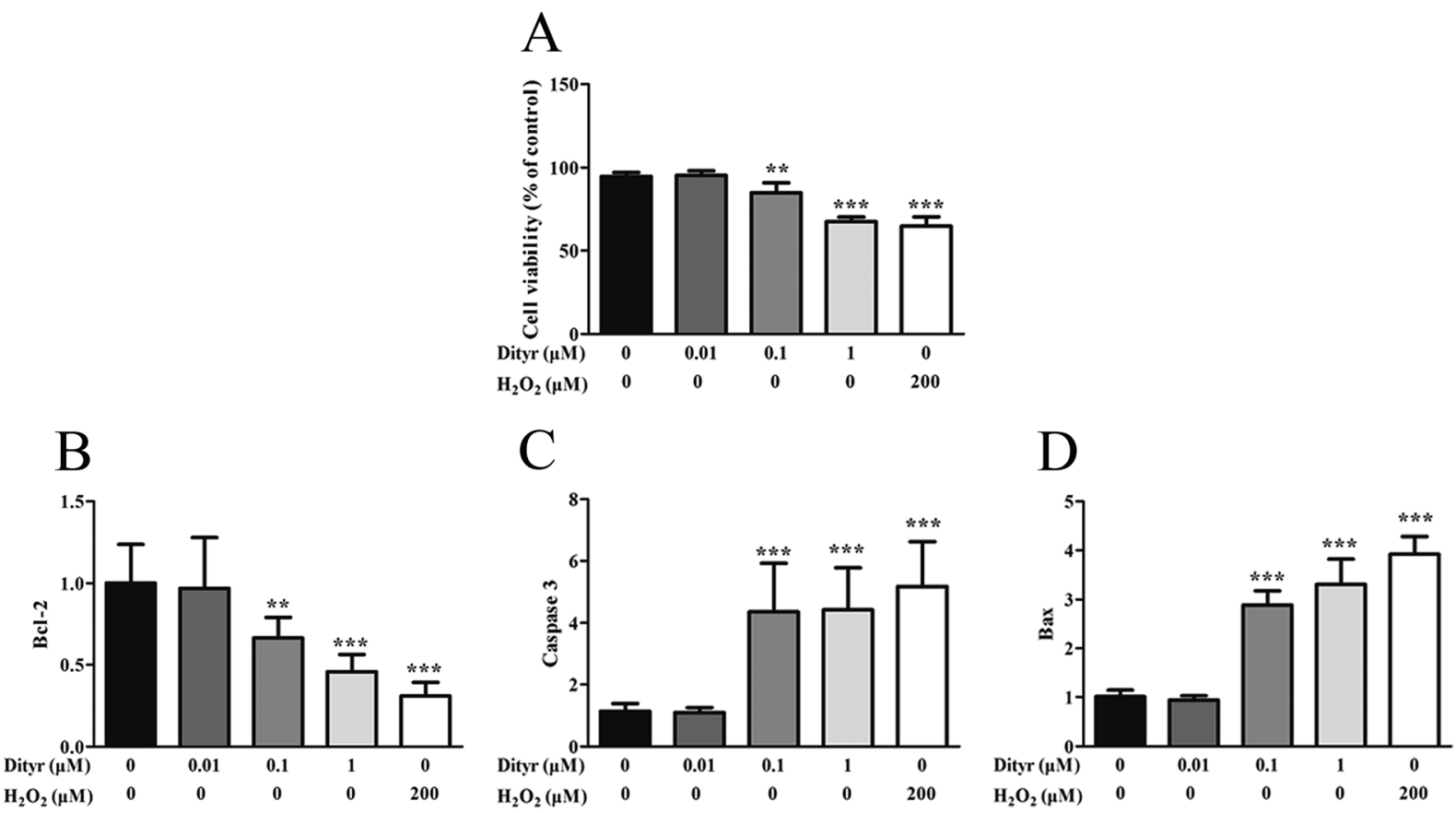

Fig. 15 Effects of Dityr on the cell viability of MIN-6 cells. (A) Dityr decreased the cell viability, which was determined by MTT. The data are expressed as percentages of untreated control cells. (B-D) Dityr downregulated the mRNA expression of genes in mitochondria-mediated apoptotic signaling pathways. (B) Bcl-2. (C) Caspase 3. (D) Bax. ${ }^{*} p<0.05 ; * * p 0.01 ; * * * p<0.001$.

activates ATP generation, which induces the closure of ATPsensitive $\mathrm{K}\left(\mathrm{K}_{\mathrm{ATP}}\right)$ channels and the depolarization of the plasma membrane, resulting in insulin release. ${ }^{51,52}$ Impaired GSIS can be induced by reduced glucose sensibility, disrupted mitochondrial metabolism and function, or a combination of both. As expected, in the present study, exposure to Dityr altered the mRNA level of Glut2 and Gck, both in MIN-6 cells and the mice pancreas. These results suggest that Dityr could impair the GSIS pathway by regulating the rate of glucose uptake and glycolysis. In addition, Dityr decreased the expression of the $\mathrm{K}_{\mathrm{ATP}}$ channel subunits Kir6.2 and Sur, which might account for the inhibition of insulin secretion in both Dityr-treated mice and cells. ${ }^{51}$ This effect was accompanied by significant downregulation of the mRNA level of subunit 6 of mitochondrial ATP synthase (ATP6). These findings indicate that Dityr-induced mitochondrial damage may play an important role in the onset of $\beta$ cell dysfunction.

In the present study, the OTPs and Dityr treatments had adverse effects on several key events involved in the regulation of mitochondrial function and metabolism in the mice pancreas and MIN-6 cells. For example, the OTPs and Dityr altered the mRNA levels of genes that were central to mitochondrial function and metabolism. Citrate synthase is a ratelimiting enzyme in the TCA cycle, OGDH encodes ratelimiting enzymes in the mitochondrial TCA cycle, and UCP2 negatively regulates the sensitivity of insulin secretion to glucose stimulation and mitochondrial ATP production in $\beta$ cells..$^{53}$ The down-regulation of ATP6, citrate synthase, and $O G D H$, in addition to the up-regulation of UCP2, likely contributes to Dityr-induced defects in ATP generation and impaired GSIS. The mitochondrial transcription factor TFAM plays a role in mitochondrial biogenesis and respiration in response to environmental stimuli. As expected, in the present study, the TFAM mRNA level was reduced in the OTP/Dityrtreated mice and Dityr-exposed cells. Previous research showed that the expression of TFAM was specifically associated with the mtDNA copy number. ${ }^{54}$

mtDNA depletion, which plays a major role in mitochondrial diseases, is another potential cause of mitochondrial dysfunction. $^{55}$ mtDNA is prone to oxidative damage. In addition to mitochondrial dysfunction, previous research showed that an abnormal mtDNA copy number was associated with increased oxidative stress. ${ }^{56,57}$ Genetically altered mice lacking expression of the mitochondrial genome in $\beta$ cells exhibited symptoms of diabetes and an impaired GSIS pathway in pancreatic islets. ${ }^{58} \mathrm{In}$ the present study, the results of the western blot and mtDNA copy number assay showed that the OTP and Dityr treatments depleted mtDNA in the mice pancreas and MIN-6 cells. Increased ROS production is associated with mtDNA damage. ${ }^{21}$ In the current study, Dityr induced excessive ROS generation both in Dityr-treated mice and MIN-6 cells. Since $\mathrm{H}_{2} \mathrm{O}_{2}$ exposure also led to increased ROS levels and decreased mtDNA copy numbers in MIN-6 cells, it suggested that Dityr-induced oxidative stress would be responsible for the mtDNA depletion.

A deficiency in mitochondrial membrane integrity may be another confounding factor of mitochondrial dysfunction..$^{59,60}$ The membrane potential is a marker of the integrity of the mitochondrial membrane. Many studies have revealed the relationship between the membrane potential and ATP production. $^{61,62}$ In the present study, mitochondrial membrane 
depolarization was observed in the Dityr-exposed MIN-6 cells and in the pancreas of the OTPs/Dityr-treated mice. These results suggested that Dityr destroyed the structural integrity of the mitochondrion. The results also demonstrated that the OTPs and Dityr treatments had a negative effect on mitochondrial function.

One potential result of oxidative stress-induced mitochondrial dysfunction is cell apoptosis. The apoptosis of pancreatic $\beta$ cells is a critical component in the pathogenesis of diabetes mellitus. ${ }^{63,64}$ In the present study, both OTPs and Dityr induced cell death via activation of the mitochondrial apoptotic pathway, including the Bcl-2 and caspase families. Bcl-2 is an anti-apoptotic factor, which plays an essential role in maintaining the integrity of the mitochondrial outer membrane. ${ }^{65}$ Previous research reported that Bcl-2 was a central regulator of the mitochondrial-dependent apoptotic pathway. ${ }^{66}$ Bax is a proapoptotic protein, which has been shown to induce mitochondrial membrane depolarization and promote the release of apoptogenic factors from mitochondria, leading to cell death. ${ }^{66}$ The caspase family plays a critical role in the release of apoptotic signal. ${ }^{67}$ The findings of the present study demonstrate that OTPs and Dityr induced down-regulation of the $\mathrm{Bcl}-2$ mRNA level, as well as up-regulation of Bax, causing altered mitochondrial membrane permeability and subsequent upregulation of Caspase 3. All the findings indicate that the mitochondrial-mediated apoptotic signalling pathway, triggered by OTPs, is involved in the apoptotic process and that Dityr may be responsible for the injury induced by OTPs.

In conclusion, the results of the present study suggest that OTP induced oxidative injury in the pancreas potentially results in the disruption of glucose metabolism. Dityr, as the main oxidized product in OTPs, is responsible for OTPs-induced pancreatic injury. Therefore, we suggest that oxidative stressinduced mitochondrial dysfunction and mitochondrial-related apoptotic signals play an essential role in the OTPs/Dityrinduced functional insufficiency of pancreatic $\beta$ cells.

\section{Conflict of interest}

The authors declare that there are no conflicts of interest.

\section{Acknowledgements}

This work was supported by the Chinese Nature Science Foundation (31571841), Natural Science Foundation of Jiangsu Providence, China (BK20140147).

\section{References}

1 M. J. Davies, Biochim. Biophys. Acta, 2005, 1703, 93-109.

2 M. Estevez, Poult. Sci., 2015, 94, 1368-1378.

3 C. Hellmuth, F. F. Kirchberg, N. Lass, U. Harder, W. Peissner, B. Koletzko and T. Reinehr, J. Diabetes Res., 2016, 2016, 2108909.

4 B. Lu, P. Gu, Y. Xu, X. Ye, Y. Wang, H. Du and J. Shao, Minerva Endocrinol., 2016, 41, 1-9.

5 M. A. Malik, S. N. Basahel, A. Y. Obaid and Z. Khan, Colloids Surf., B, 2010, 76, 346-353.
6 Y. Fukuchi, Y. Miura, Y. Nabeno, Y. Kato, T. Osawa and M. Naito, J. Atheroscler. Thromb., 2008, 15, 185-192.

7 D. A. Malencik and S. R. Anderson, Amino Acids, 2003, 25, 233-247.

8 A. Tonelli, M. G. D'Angelo, R. Salati, L. Villa, C. Germinasi, T. Frattini, G. Meola, A. C. Turconi, N. Bresolin and M. T. Bassi, J. Neurol. Sci., 2006, 241, 13-17.

9 M. Abdolvahab, K. Behnaz, B. Sima and M. Teimoorian, J. Global Infect. Dis., 2010, 2, 198-199.

10 D. A. Butterfield, M. Perluigi and R. Sultana, Eur. J. Pharmacol., 2006, 545, 39-50.

11 C. F. Lin, A. Asghar, J. I. Gray, D. J. Buckley, A. M. Booren, R. L. Crackel and C. J. Flegal, Br. Poult. Sci., 1989, 30, 855-864.

12 A. Beyerlein and R. von Kries, Am. J. Clin. Nutr., 2011, 94, 1772S-1775S.

13 M. M. El-Shafei, N. S. Al-Amoudy and A. K. Said, Die Nahrung, 1988, 32, 559-564.

14 Z. L. Li, L. Mo, G. Le and Y. Shi, Food Chem. Toxicol., 2014, 64, 86-93.

15 L. Zhuqing Leslie, Y. Shi, G. Le, Y. Ding and Q. Zhao, Oxid. Med. Cell. Longevity, 2016, 1, 1-12.

16 Y. Ran, B. Yan, Z. Li, Y. Ding, Y. Shi and G. Le, Physiol. Behav., 2016, 164, 292-299.

17 X. G. Lei and M. Z. Vatamaniuk, Antioxid. Redox Signaling, 2011, 14, 489-503.

18 V. Ramakrishna and R. Jailkhani, Acta Diabetol., 2008, 45, 41-46.

19 B. B. Lowell and G. I. Shulman, Science, 2005, 307, 384-387.

20 J. Szendroedi, E. Phielix and M. Roden, Nat. Rev. Endocrinol., 2012, 8, 92-103.

21 P. Maechler and P. B. de Andrade, Biochem. Soc. Trans., 2006, 34, 824-827.

22 E. K. Fetisova, A. V. Avetisyan, D. S. Izyumov, M. V. Korotetskaya, B. V. Chernyak and V. P. Skulachev, FEBS Lett., 2010, 584, 562-566.

23 P. F. Wu, L. H. Long, J. H. Zeng, X. L. Guan, J. Zhou, Y. Jin, L. Ni, F. Wang, J. G. Chen and N. Xie, Food Chem. Toxicol., 2012, 50, 2729-2735.

24 R. Haenold, R. Wassef, N. Brot, S. Neugebauer, E. Leipold, S. H. Heinemann and T. Hoshi, Free Radical Res., 2008, 42, 978-988.

25 P. Maechler and C. B. Wollheim, Nature, 2001, 414, 807-812. 26 Z. L. Li, Y. Shi, Y. Ding, Y. Ran and G. Le, Amino Acids, 2016, 1-20.

27 T. Kurahashi, A. Miyazaki, S. Suwan and M. Isobe, J. Am. Chem. Soc., 2001, 123, 9268-9278.

28 O. H. Lowry, N. J. Rosebrough, A. L. Farr and R. J. Randall, J. Biol. Chem., 1951, 193, 265-275.

29 V. Venegas, J. Wang, D. Dimmock and L. J. Wong, et al., Current protocols in human genetics/editorial board, $\mathrm{L}$. Jonathan Haines, 2011, Ch. 19, Unit 1917.

30 T. K. Dalsgaard, J. H. Nielsen, B. E. Brown, N. Stadler and M. J. Davies, J. Agric. Food Chem., 2011, 59, 7939-7947.

31 E. R. Stadtman, Annu. Rev. Biochem., 1993, 62, 797-821.

32 Z. L. Li, Y. Shi, G. Le, Y. Ding and Q. Zhao, Oxid. Med. Cell. Longevity, 2016, 2016, 3123294. 
33 M. Tiedge, S. Lortz, J. Drinkgern and S. Lenzen, Diabetes, 1997, 46, 1733-1742.

34 V. Poitout and R. P. Robertson, Endocr. Rev., 2008, 29, 351366.

35 N. Bashan, J. Kovsan, I. Kachko, H. Ovadia and A. Rudich, Physiol. Rev., 2009, 89, 27-71.

36 D. P. Jones, Methods Enzymol., 2002, 348, 93-112.

37 S. Kwan Tat, M. Padrines, S. Theoleyre, D. Heymann and Y. Fortun, Cytokine Growth Factor Rev., 2004, 15, 49-60.

38 S. M. Fathy and E. A. Drees, BMC Complementary Altern. Med., 2016, 16, 8.

39 W. Yin, B. Li, X. Li, F. Yu, Q. Cai, Z. Zhang, M. Cheng and H. Gao, Food Funct., 2015, 6, 3065-3071.

40 D. V. Chartoumpekis and T. W. Kensler, Curr. Diabetes Rev., 2013, 9, 137-145.

41 H. Motohashi and M. Yamamoto, Trends Mol. Med., 2004, 10, 549-557.

42 G. E. Mann, J. Niehueser-Saran, A. Watson, L. Gao, T. Ishii, P. de Winter and R. C. Siow, Acta Physiol. Sin., 2007, 59, 117-127.

43 J. Ryu, R. Zhang, B. H. Hong, E. J. Yang, K. A. Kang, M. Choi, K. C. Kim, S. J. Noh, H. S. Kim, N. H. Lee, J. W. Hyun and H. S. Kim, PLoS One, 2013, 8, e71178.

44 B. C. Scott, O. I. Aruoma, P. J. Evans, C. O'Neill, A. Van der Vliet, C. E. Cross, H. Tritschler and B. Halliwell, Free Radical Res., 1994, 20, 119-133.

45 L. Rochette, S. Ghibu, A. Muresan and C. Vergely, Can. J. Physiol. Pharmacol., 2015, 93, 1021-1027.

46 R. M. Ogborne, S. A. Rushworth and M. A. O'Connell, Arterioscler., Thromb., Vasc. Biol., 2005, 25, 2100-2105.

47 J. H. Choi, S. O. Cho and H. Kim, Yonsei Med. J., 2016, 57, 260-264.

48 B. Lipinski, Oncology, 2001, 60, 373.

49 P. F. Chinnery and E. A. Schon, J. Neurol., Neurosurg. Psychiatry, 2003, 74, 1188-1199.

50 A. Navarro and A. Boveris, Am. J. Physiol., 2007, 292, C670C686.

51 J. C. Henquin, Diabetologia, 2009, 52, 739-751.

52 P. Rorsman and E. Renstrom, Diabetologia, 2003, 46, 10291045.
53 C. B. Chan, D. De Leo, J. W. Joseph, T. S. McQuaid, X. F. Ha, F. Xu, R. G. Tsushima, P. S. Pennefather, A. M. Salapatek and M. B. Wheeler, Diabetes, 2001, 50, 1302-1310.

54 M. I. Ekstrand, M. Falkenberg, A. Rantanen, C. B. Park, M. Gaspari, K. Hultenby, P. Rustin, C. M. Gustafsson and N. G. Larsson, Hum. Mol. Genet., 2004, 13, 935-944.

55 L. J. Wong, R. K. Naviaux, N. Brunetti-Pierri, Q. Zhang, E. S. Schmitt, C. Truong, M. Milone, B. H. Cohen, B. Wical, J. Ganesh, A. A. Basinger, B. K. Burton, K. Swoboda, D. L. Gilbert, A. Vanderver, R. P. Saneto, B. Maranda, G. Arnold, J. E. Abdenur, P. J. Waters and W. C. Copeland, Hum. Mutat., 2008, 29, E150-E172.

56 S. Yamada, S. Nomoto, T. Fujii, T. Kaneko, S. Takeda, S. Inoue, N. Kanazumi and A. Nakao, Eur. J. Surg. Oncol., 2006, 32, 303-307.

57 A. N. Malik and A. Czajka, Mitochondrion, 2013, 13, 481-492. 58 J. P. Silva, M. Kohler, C. Graff, A. Oldfors, M. A. Magnuson, P. O. Berggren and N. G. Larsson, Nat. Genet., 2000, 26, 336-340.

59 A. Jelinek and H. P. Klocking, Exp. Toxicol. Pathol., 1998, 50, 472-476.

60 M. Darshi, V. L. Mendiola, M. R. Mackey, A. N. Murphy, A. Koller, G. A. Perkins, M. H. Ellisman and S. S. Taylor, J. Biol. Chem., 2011, 286, 2918-2932.

61 A. Vojtiskova, P. Jesina, M. Kalous, V. Kaplanova, J. Houstek, M. Tesarova, D. Fornuskova, J. Zeman, A. Dubot and C. Godinot, Toxicol. Mech. Methods, 2004, 14, 7-11.

62 F. Distelmaier, H. J. Visch, J. A. Smeitink, E. Mayatepek, W. J. Koopman and P. H. Willems, J. Mol. Med., 2009, 87, 515-522.

63 B. Faideau, E. Larger, F. Lepault, J. C. Carel and C. Boitard, Diabetes, 2005, 54(Suppl 2), S87-S96.

64 C. M. Filippi and M. G. von Herrath, Diabetes, 2008, 57, 2863-2871.

65 G. Kroemer and J. C. Reed, Nat. Med., 2000, 6, 513-519.

66 R. J. Youle and A. Strasser, Nat. Rev. Mol. Cell Biol., 2008, 9, 47-59.

67 W. C. Earnshaw, L. M. Martins and S. H. Kaufmann, Annu. Rev. Biochem., 1999, 68, 383-424. 